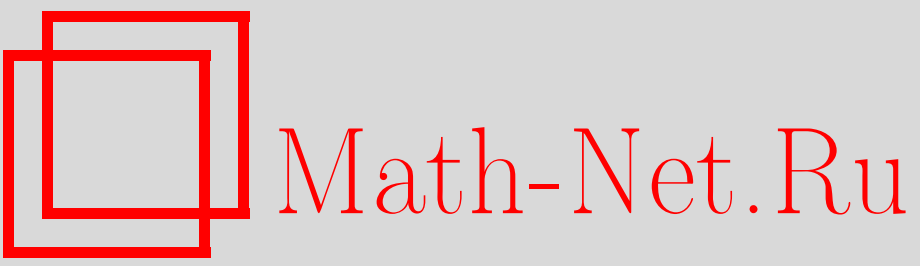

В. А. Малышев, Вероятность вокруг квантовой гравитации: планарная гравитация, УМН, 1999, том 54, выпуск 4, 3-46

DOI: https://doi.org/10.4213/rm178

Использование Общероссийского математического портала Math-Net.Ru подразумевает, что вы прочитали и согласны с пользовательским соглашением

http://www . mathnet.ru/rus/agreement

Параметры загрузки:

IP: 54.147 .182 .235

26 апреля 2023 г., 14:06:08 


\title{
ВЕРОЯТНОСТЬ ВОКРУГ КВАНТОВОЙ ГРАВИТАЦИИ: ПЛАНАРНАЯ ГРАВИТАЦИЯ
}

\author{
В. А. МАлышев
}

Изучается стохастическая динамика, оставляющая инвариантной равновесное распределение квантовой гравитации. Это первое развернутое теоретическое изучение такой динамики (ранее она использовалась для численного моделирования методами Монте-Карло). Основной результат касается существования и свойств локальных корреляционных функций в термодинамическом пределе. В то же время эта работа может служить математическим введением в квантовую гравитацию: мы даем строгое изложение квантовой гравитации для плоской чистой гравитации. В основном мы используем комбинаторный подход вместо более популярных в физике матричных моделей, в центре изложения - знаменитая экспонента $\alpha=-\frac{7}{2}$.

Библиография: 32 названия.

\section{СОДЕРЖАНИЕ}

1. Введение

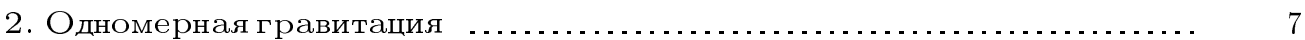

2.1. Равновесное распределение ............................... 8

2.2. Гравитация как модель теории очередей ........................ 10

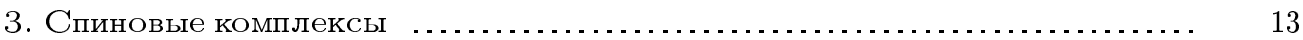

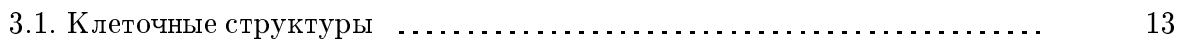

3.1.1. Абстрактные комплексы .............................. 13

3.1.2. Топологические комплексы . ............................. 13

3.1.3. Клеточные поверхности . . . . . . . . . . . . . . . . . . . . . . . . . . . 14

3.1.4. Локальный наблюдатель (корень) … . . . . . . . . . . . . . . . . . 15

3.1.5. Движения ............................................. 16

3.1.6. Группа автоморфизмов ............................. 17

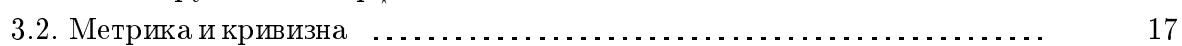

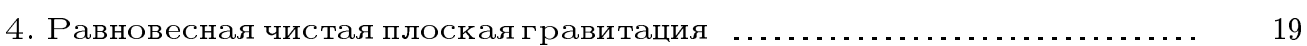

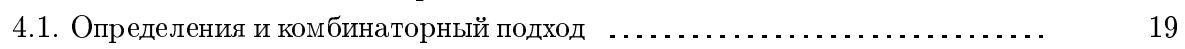

4.1.1. Равномерная асимптотика ............................. 24

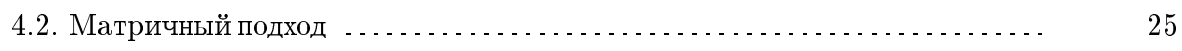

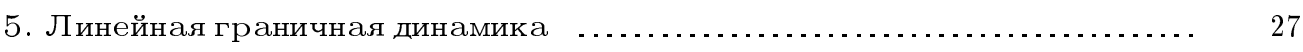

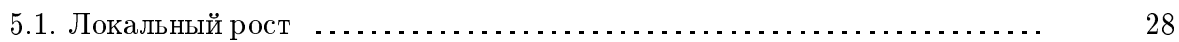

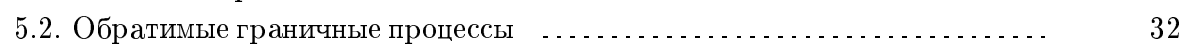

6. Нелинейная граничная динамика ............................. 32 


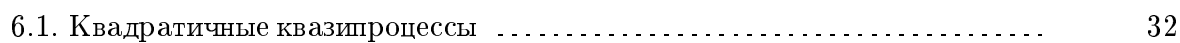

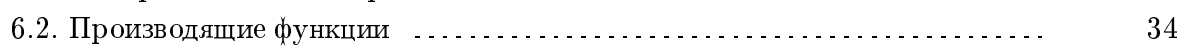

6.2.1. Анализ функционального уравнения ....................... 36

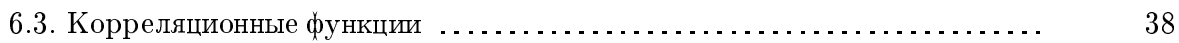

6.3.1. Представление комплексов деревьями ..................... 38

6.3.2. Локальная кривизна … . . . . .

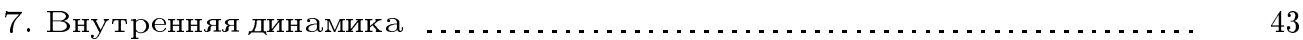

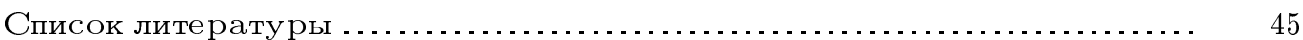

\section{1. Введение}

Немного истории. Следует сказать несколько слов об истории квантовой гравитации. Классическая гравитация имеет дело с гладким (не обязательно четырехмерным) многообразием $M$, псевдометрикой $g_{i j}$ на нем и с действием Гильберта-Эйнштейна

$$
S=\int_{M}(\lambda R(x)+L(x)+\mu) \sqrt{\operatorname{det} g} d x
$$

стационарные точки которого и изучаются. Здесь $R(x)$ - внутренняя кривизна в точке $x, L$ - некоторьй функционал от полей $\phi(x)$, соответствуюших вешеству, $\mu$ - космологическая константа. В чистой (без вешества) гравитации $L=0$, и здесь рассматривается исключительно этот случай. Квантовая гравитация принимает во внимание не только стационарные точки, но также все остальные конфигурации, приписьвая им некоторые веса. Иначе говоря, вводится формальная плотность (которая становится положительной при переходе к евклидовой метрике)

$$
Z^{-1} \exp (-\mu S)
$$

на некотором пространстве конфигураций $\Omega$ полей вешества и метрических тензоров. Все ранние попытки избавиться от расходимостей привели к заключению, что $\Omega$ должно включать, помимо того, и все гладкие структуры на $M$ и даже само $M$, т.е. пространство и его топология должны быть случайными. В настоящее время единственно возможным для реализации этой программы представляется начинать с рассматрения только дискретных объектов, а затем совершать предельные переходы с одновременным скейлингом. Иначе говоря, пространство становится конечным комплексом, гладкая структура - кусочно-линейной, метрика и кривизна кодируются одно- и двумерньми скелетами комплекса, поля вещества переходят в спины, живущие на клетках комлекса. Оказьвается, что такое квантование-дискретизация применимо также к совсем другим физическим системам: релятивистской частице, струне и т. д., но с другой интерпретацией. Например, квантованная таким способом струна становится двумерным комплексом, спин в данной вершине представлен вектором из $\mathbb{R}^{d}$. Спин и осуществляет отображение вершин комплекса в $\mathbb{R}^{d}$, аппроксимируя классическую струну.

Дискретизация классической гравитации впервые рассматривалась Редже в [1], где он ввел точные определения в дискретном случае: конечное пространство-время, его кривизну и действие Гильберта-Эйнштейна. Его теория была уже включена в фундаментальную монографию [2], но в семидесятых это рассматривалось еще как отклонение от главных направлений в физике и ему посвящались только редкие работы. 
Среди таких работ была однако и известная работа С. Хокинга [3], где обсуждалась уже дискретная квантовая гравитация.

В восьмидесятые годы набирается уже более 100 работ, посвященных дискретной квантовой гравитации.

В девяностые число их переваливает за 1000 и продолжает расти. Это связано, в частности, с многочисленными связями дискретной гравитации как с другими современными математическими, так и с физическими теориями такими, как струны, случайные матрищы и другое. Более того, современные статьи по теоретической физике часто содержат следуюшие сентенции: “Двумерная случайная геометрия становится в центр многих моделей современной физики, от теории струн и двумерной квантовой гравитации, в которых делаются попытки описать фундаментальные взаимодействия, до мембран и межфазовых флуктуаций в многочисленных задачах физики конденсированного состояния", см. [4].

Для вероятностника квантовая гравитация является источником вдохновения, новой математики и философии в теории вероятностей. Статья может служить математическим введением в дискретную квантовую гравитацию для специалистов по теории вероятностей. Следует сказать, однако, что предмет квантовой гравитации гораздо шире, о различных ее аспектах см. обзор Ровелли [5].

Динамика против равновесия. В основном мы используем комбинаторный подход, вместо более популярного в физике подхода, основанного на случайных матричных моделях (CMM). Однако центром притяжения в нашей статье, как и в физике, является знаменитая экспонента $\alpha=-\frac{7}{2}$. Другой целью статьи является рассмотрение стохастической динамики, оставляющей равновесное распределение, рассматриваемое в квантовой гравитации, инвариантньм. Здесь мы начинаем теоретическое исследование такой динамики (ранее она использовалась лишь для моделирования методом Монте-Карло; изучение динамики может рассматриваться как третья часть в серии работ автора (см. [6], [7]), но знание предыдущих статей не требуется для чтения данной статьи). Дело в том, что подобные процессы имеют универсальный характер и охватывают большинство конкретных марковских процессов, имея много аналогов в информатике и биологии.

Под вероятностью мы понимаем здесь классическую теорию вероятностей. Квантовую гравитацию образуют сейчас множество работ, переполняющих в последние 10 лет хорошо известные физические журналы. Дискретная квантовая гравитация рассматривается сейчас как многообешающее направление для объединения максимальной и минимальной пространственно-временных шкал в сушествуюшей картине природы. Мы ограничиваемся частью этой области, в которой используется вероятностная интуиция, однако трудно найти даже точные формулировки, не говоря о доказательствах, которые были бы удовлетворительны для математика: даже если вероятности положительны, они ненормированы. Кажется, что это определяется не пренебрежением авторов к подобным мелочам, а более глубокими причинами.

В физической литературе алгеброгеометрическая техника довлеет над всей областью. Поэтому полезно обсудить простейшие вероятностные аспекты квантовой гравитации: даже такая попытка немедленно порождает много естественных вопросов.

Сейчас известны два подхода в дискретной квантовой гравитации: квантовое исчисление Редже (в котором длины ребер являются случайными переменными) и динамические триангуляции (где длины ребер одинаковы). Термин “динамические" во 
втором подходе может ввести в заблуждение, так как в действительности в этом подходе нет никакой динамики: основная техника использует распределения Гиббса на множестве больших случайных матриц. Поэтому здесь мы называем эти подходы равновесными.

Динамика в квантовой гравитации появилась в моделировании по Монте-Карло. Новое в данной статье - это аналитическое и вероятностное изучение подобных процессов (автору неизвестны более ранние строгие результаты). Почему такое изучение может быть полезным? Этому есть много причин - мы даем неполный список.

- Хорошо известная трудность в усреднении по всем топологиям состоит в том, что, в размерности 4, это порождает алгоритмически неразрешимые проблемы. Динамика заменяет эту проблему другой: вместо усреднения мы ищем процесс (с произвольным начальным состоянием), который порождает некоторьй класс топологий. Он должен иметь некоторые симметрии, но также должен быть законным с точки зрения теории вероятностей, и уже здесь возникают интересные наблюдения.

- Как при стохастическом квантовании в обычной квантовой теории поля и глауберовой динамике в статистической механике, хотелось бы иметь марковский процесс, оставляющий инвариантной гиббсовскую меру. Эта динамика полезна (но не фундаментальна) в квантовой теории поля, где однако есть аксиомы Уайтмана, и в статистической механике, где есть исконная детерминистическая динамика Ньютона, которая рассматривается как более фундаментальная, чем сама мера Гиббса. В квантовой гравитации, где нет ни того ни другого, можно обратиться к неожиданной точке зрения, что сам процесс является более фундаментальным, чем сама мера Гиббса.

- Динамика позволяет рассматривать область ниже критической точки, где равновесное распределение не имеет смысла. И с вероятностной точки зрения эта область представляется не менее естественной - как растушая Вселенная (в компюютерном времени - термин, который автору известен из статьи А. Мигдала). Более того, динамика придает смысл величинам в критической точке без перехода к скейлинговому пределу. Автору не известна истинная физическая интерпретация этого, но естественность с вероятностной точки зрения более чем очевидна.

- У нас нет никаких физических аргументов за динамические модели (или против них), и ведущей нитью может быть только вероятностная интуиция. Главные вопросы: что такое универсальность и ситуация общего положения? Недавно было предположено, см. [8], что информатика может играть роль в будущих физических теориях. Динамический подход позволяет интерпретировать эволюцию Вселенной как случайную грамматику, описывающую случайную эволюцию языка.

- Математические работы по термодинамическому переходу в статистической механике и квантовой теории поля принесли много новых идей в теорию вероятностей. Но попытка построить подобную теорию для растущих случайных комплексов приносит дополнительные неизвестные ранее явления (см. [6], [7]), возможно имеющие и физическую интерпретацию. Один из таких новых эффектов заключается в том, что невозможно фиксировать точку отсчета (начало координат) в бесконечной Вселенной без использования аксиомы Цермело, а 
любое конструктивное введение локального наблюдателя в систему изменяет структуру пространства-времени в его окрестности.

- С помощью моделирования на компютере по методу Монте-Карло трудно найти критические экспоненты, поскольку в асимптотике доминирует экспоненциальньй множитель, которьй не универсален (сильно зависит от деталей модели). Более полезным, чем моделирование равномерного распределения на множестве триангулящий с заданньм числом клеток, для этого был бы марковский процесс, меняющий число клеток. Оказывается, что для нужной экспоненты $\alpha=-\frac{7}{2}$ естественным является не обычньй марковский процесс, а некоторое преобразование мер, называемое в теории вероятностей нелинейным марковским процессом.

Содержание работы. В разделе 2 рассматривается одномерный случай, который полезен как мост, соединяющий классическую вероятность, например, теорию массового обслуживания, с двумерной квантовой гравитацией.

В разделе 3 дается минимум необходимых определений относительно комплексов и кривизны в двумерном случае.

Раздел 4 содержит вводные определения, основные задачи и некоторые известные результаты. В разделе 4.2 дается краткое изложение подхода СММ к чистой плоской гравитации, единственной целью которого является подчеркнуть некоторые вопросы, связанные с комбинаторным подходом, основньм в этой статье.

В разделе 5 изучается простая динамическая модель, где клетки добавляются случайно к границе диска. Эта модель явно решаема (с помошью случайных блужданий), и мы выгисляем основные величины. Экспонента в этой модели равна -2 и принадлежит, таким образом, к универсальному классу, отличному от принятого в физике. Однако непрерывный предел в этой модели также определен, что дает пространство постоянной кривизны.

Раздел 6 является центральным. Здесь строятся и изучаются нелинейные марковские процессы, оставляющие меру Гиббса инвариантной. В них также замены могут быть только на границе. Мы используем метод функциональных уравнений Тутте для получения нужной экспоненты $-\frac{7}{2}$. С другой стороны, мы развиваем и новьй подход (комбинаторный) для изучения локальных коррелящионных функций.

В разделе 7 рассматривается марковская динамика, где замены могут быть на любой клетке комплекса.

Я благодарю Л. Пастура, которьй прояснил мне некоторые моменты в теории случайных матриц, а также С. Шлосмана и Р. Минлоса за прочтение работы и множество полезных замечаний.

\section{2. Одномерная гравитация}

По поводу физических аргументов и красивых вычислений в одномерной гравитации мы отсылаем к главе 2 лекций Амбьерна [9]. Нашей целью является вероятностное рассмотрение гравитации и обсуждение неожиданных связей. В одномерном случае нет топологии: одномерньй комплекс является линейным графоом.

Цепочка символов из некоторого алфавита может рассматриваться как функция на вершинах этого графа (см. рис. 1). 


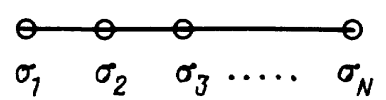

Рис. 1. Линейный спиновый графф

2.1. Равновесное распределение. Мы дадим основное определение в более абстрактном виде, чем в [9], без предварительного вложения в евклидово пространство: это более соответствует квантованию струн по Полякову. Рассмотрим вероятностные распределения на множестве конечных линейных спин-графов (иногда мы пользуемся терминологией из [6], [7], но такие отступления можно опускать без ушерба для понимания). Иначе говоря, рассмотрим распределения на множестве $\Omega$ цепочек $\sigma=s_{1} \ldots s_{N}$, где $N=0,1,2, \ldots$ и $s_{i} \in S$, где $S$ - алфавит (множество спинов). Например, $S$ может быть единичной сферой в $\mathbb{Z}^{d}$ или в $\mathbb{R}^{d}$. Случай $N=0$ соответствует пустой цепочке, которой не приписьвается никакого $S$-значения. Определим неотрицательную меру на $\Omega$

$$
Q(\sigma)=\exp \left(-\mu N-\beta \sum_{i=0}^{N-1}\left(f_{1}\left(s_{i+1}\right)+f_{2}\left(s_{i}, s_{i+1}\right)\right)\right)
$$

для некоторых функций $f_{1}, f_{2}$. Удобно предположить, что фиксирован некоторьй элемент $s_{0}$. Простейший пример получается, если $s \in \mathbb{Z}^{d}, f_{2}=0, f_{1}(s)=\infty$ для всех $s$ кроме конечного числа. Эта мера может быть нормирована, если

$$
Z=\sum_{N, \sigma} Q(N, \sigma)<\infty
$$

Интуитивно, последовательность Значений $s_{0}, s_{0}+s_{1}, \ldots, s_{0}+s_{1}+\cdots+s_{N}$ может рассматриваться как случайное блуждание. Однако оно отлично от классических случайных блужданий. Мы увидим ниже его связь с некоторыми задачами информатики. Этот формальньй объект затем будет привязан к евклидову пространству, в физике также принята сейчас подобная процедура - абстрактньй объект (случайная триангуляция, внутренняя метрика и т. д.) привязывается к физическому пространству-времени.

Простейшие примеры. В первом примере $S=\mathbb{Z}^{d}, f_{2} \equiv 0, f_{1}(s)=1$, если $|s|=1$, и $\infty$ в остальных случаях, пусть $s_{0}=0$. Иначе говоря, рассмотрим ненормированные меры $\exp (-l(s))$ на всех конечных путях $r=\left(0, s_{1}, s_{1}+s_{2}, \ldots, s_{1}+s_{2}+\cdots+s_{N}\right)$ в $\mathbb{Z}^{d}$, начинаюшихся из 0 , где $N=l(r)$ - длина (число шагов) $r$. Они не всегда конечны. Существует $0<\mu_{\mathrm{cr}}<\infty$ такое, что ряд

$$
Z=\sum_{r} \exp (-\mu l(r))
$$

для статистической суммы сходится при $\mu>\mu_{\mathrm{cr}}$ и расходится при $\mu \leqslant \mu_{\mathrm{cr}}$. В нашем случае $\mu_{\mathrm{cr}}=\ln 2 d$.

Во втором примере $S=\mathbb{R}^{d}, e^{-f_{1}(s)}=\delta(|s|-1), f_{2}\left(s, s^{\prime}\right)=\phi\left(\left(s, s^{\prime}\right)\right)$ для некоторой ограниченной функции $\phi$ от угла между двумя векторами. Для третьего примера $S=\mathbb{R}^{d}, f_{2} \equiv 0, f_{1}(s)=s^{2}=\left(s^{1}\right)^{2}+\cdots+\left(s^{d}\right)^{2}$. 
Эти примеры, весьма простые и не содержащие ничего нового с вероятностной точки зрения, соответствуют одномерньм аналогам довольно знаменитых функционалов действия в физике: действию свободной релятивистской частицы в $d$-мерном пространстве-времени $(l-$ длина, $L=L(0, x)$ - путь из 0 в $x)$

$$
S(L)=\mu \int_{L} d l
$$

действию Гильберта-Эйнштейна ( $\kappa$ - кривизна кривой, вложенной в евклидово пространство)

$$
S(L)=\mu \int_{L} d l+\lambda \int_{L}|\kappa| d l
$$

и действию для бозонной струны $x=x(l)(g$-некоторая метрика на интервале изменения параметра)

$$
S(L)=\frac{1}{\alpha^{\prime}} \int_{L} \sqrt{g(l)} d l\left(g(l) \frac{d^{2} x}{d l^{2}}+\mu\right) .
$$

Можно рассматривать введенное распределение как квантование соответствующего классического действия, оно, кстати, совпадает с дискретизацией. Каждому ребру дискретного пути приписьвается единичная длина, и тогда длина пути равна числу ребер.

Мы обсудим только первый пример, остальные рассматриваются аналогично,

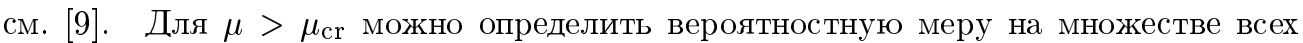
конечных путей, стартующих из нуля 0 :

$$
P(r)=Z^{-1} \exp (-\mu l(r)) .
$$

Функции Грина определяются как меры на $\mathbb{Z}^{d}$ :

$$
G(x)=\sum_{s: 0 \rightarrow x} \exp (-\mu l(r))=\sum_{N} C(N ; x) \exp (-\mu N)=\sum_{N} P_{R W}^{(N)}(x) e^{\left(-\mu+\mu_{\mathrm{cr}}\right) N},
$$

где $C(N ; x)$ - число путей из 0 в $x$ длины $N, P_{R W}^{(N)}(x)$ - вероятности перехода из 0 в $x$ за $N$ шагов для классического случайного блуждания на $\mathbb{Z}^{d}$. Число таких путей равно (по локальной предельной теореме)

$$
C(N ; x) \sim(2 \pi)^{-\frac{d}{2}}(2 d)^{N} N^{-\frac{d}{2}},
$$

если $x=O(\sqrt{N})$. Сам термин “функции Грина" идет не только от физических функций Грина, но и от функций Грина марковских процессов. Например, для случайного блуждания

$$
G_{R W}(0, x)=\sum_{n} p_{0 x}^{(n)}=\sum_{L: 0 \rightarrow x} p(L),
$$

где $L$ - все пути из 0 в $x$.

Следуюшие наблюдения важны:

- $\mu_{\mathrm{cr}}$ и $-\frac{d}{2}$ не зависят от выбора $x$; 
- $\mu_{\mathrm{cr}}$ не универсальна, она зависит от размерности и от вида решетки; также другие значения $\mu_{\mathrm{cr}}$ получатся, если рассматривать кусочно-линейные пути в $\mathbb{R}^{d}$ со сторонами фиксированной длины (см. [9]);

- однако экспонента - $\frac{d}{2}$ не зависит от формы решетки в $\mathbb{R}^{d}$, что немедленно следует из локальной предельной теоремы;

- для $\mu=\mu_{\mathrm{cr}}$ ряд сходится, если $d>2$.

Мы хотим изучить скейлинговый предел $\mu \rightarrow \mu_{\mathrm{cr}}+0$. Обозначим $x=s_{1}+\cdots+s_{N}$. Точнее, мы подсчитаем следующие экспоненты в скейлинговом пределе: массу (обратная корреляционная длина) $\nu$, экспоненту восприимчивости $\gamma$, аномальную размерность $\eta$, размерность Хаусдорфа $d_{H}$. Они определяются ведушим членом в асимптотике для малых $\mu-\mu_{\mathrm{cr}}$ :

$$
\begin{gathered}
m(\mu) \approx\left(\mu-\mu_{\mathrm{cr}}\right)^{\nu}, \quad \sum_{x} G(x) \approx\left(\mu-\mu_{\mathrm{cr}}\right)^{-\gamma} \\
G(x) \approx|x|^{-d+2-\eta}, \quad \sum N C(N ; x) \exp (-\mu N) \approx x^{d_{H}} .
\end{gathered}
$$

Дадим подробный вывод. Восприимчивость

$$
\chi(\mu)=\sum_{x} G(x)=\sum_{N} e^{\left(-\mu+\mu_{\mathrm{cr}}\right) N}=\frac{1}{1-\exp \left(-\mu+\mu_{\mathrm{cr}}\right)}
$$

не зависит от размерности спина $s$ и такова же для общих спинов и взаимодействий (однако, не всегда). Тогда $\gamma=1$.

Если $\left(\mu-\mu_{\mathrm{cr}}\right) x \ll 1$, то $G(x) \approx G_{R W}(0, x)$ - функции Грина для случайного блуждания. Поэтому $\eta=0$.

Пусть $P_{R W}^{(N)}(x)$ - переходные вероятности для простого случайного блуждания на $\mathbb{Z}^{d}$ и $f(p, z)=\sum_{N} \sum_{x} P_{R W}^{(N)}(x) e^{i p x} z^{N}, z \in \mathbb{C},-$ их производяшая функция. Тогда

$$
\begin{aligned}
G(p)=f\left(p, e^{\mu_{\mathrm{cr}}-\mu}\right) & =\sum_{N} e^{\left(-\mu+\mu_{\mathrm{cr}}\right) N}\left(\frac{1}{d} \sum \cos p_{i}\right)^{N}=\frac{1}{1-e^{-\mu+\mu_{\mathrm{cr}} \frac{1}{d} \sum \cos p_{i}}} \\
& \approx \frac{1}{\mu-\mu_{\mathrm{cr}}+\frac{1}{d} \sum \cos p_{i}},
\end{aligned}
$$

что дает пропагатор свободной квантовой релятивистской частицы массы $\sqrt{\mu-\mu_{\mathrm{cr}}}$. Таким образом, $\nu=\frac{1}{2}$.

Нетрудно доказать также, что

$$
d_{H}=\nu^{-1}, \quad \gamma=\nu(2-\eta)
$$

Во втором примере другие экспоненты получаются при $\lambda \rightarrow \infty, \mu \rightarrow \mu_{\mathrm{cr}}$ (см. [9]).

2.2. Гравитация как модель теории очередей. Начнем с первого примера, приведенного выше. Можно построить обратимую динамику, сохраняющую меру $Q(\sigma)$. Это будет марковская цепь с непрерьвным временем. В нашем случае $S$ состоит из $2 d$ единичных векторов направлений $e_{1},-e_{1}, \ldots, e_{d},-e_{d}$ в $\mathbb{Z}^{d}$. Состояние

$$
\sigma=s_{1} \ldots s_{N}
$$


интерпретируется как очередь, где $N$ - ее длина, $S$ - множество типов клиентов, а $s_{1}+\cdots+s_{N} \in \mathbb{R}^{d}$ - обобшенная длина очереди. Это LIFO очередь (последний пришел, первый вьшел), и переходы состоят в добавлении и удалении крайнего справа ребра (что аналогично прибытию и обслуживанию клиента). Точнее, для всех $\sigma=s_{1} \ldots s_{N}$ с интенсивностью $\nu$

$$
s_{1} \ldots s_{N} \rightarrow s_{1} \ldots s_{N-1}
$$

а с интенсивностью $\lambda$

$$
s_{1} \ldots s_{N} \rightarrow s_{1} \ldots s_{N} s_{N+1},
$$

где значения $s_{N+1}$ выбираются равновероятно. Переходы $\varnothing \rightarrow s_{1}$ от пустой очереди имеют интенсивность $\lambda$ с равновероятными $s_{1}$.

ЛЕмма 1. Процесс әргодичен тогда и только тогда, когда $\nu>\lambda$, а распределение (1) инвариантно относительно этой динамики.

Для доказательства заметим, что длина очереди $N_{t}=N(\sigma(t))$ есть также цепь Маркова - процесс рождения-гибели, и его стационарные вероятности суть

$$
Z^{-1} \exp (-\mu N-N \ln 2), \quad \mu=\ln \frac{\nu}{\lambda} .
$$

Заметим, что система двух очередей соответствует двум взаимодействующим частицам и т. д., но мы не углубляемся в этот вопрос.

Суперкритический случай. Хотя в случае $\nu \leqslant \lambda$ нет равновесного распределения, но в динамике он ничем не хуже случая $\nu>\lambda$ : в любой момент времени сушествует распределение $Q(t)$ и можно изучать его локальные корреляционные функции при $t \rightarrow$ $\infty$. Обозначим $N(\sigma(t))$ длину цепочки $\sigma(t)$ в момент $t$.

Теорема 1. Если $\lambda>\nu$, то $N(\sigma(t)) \rightarrow \infty$ n.н. Более того, существуют предельные локальные корреляиионные функции (не слишком близко к кониам цепочки), которые определяют трансляционно инвариантную меру Гиббса на $\mathbb{Z}$. Например, одночастичная корреляционная функиия определяется так:

$$
\lim _{k \rightarrow \infty} \lim _{t \rightarrow \infty} P\left(s_{k}(t)=i\right) \rightarrow p_{i}, \quad i \in S
$$

В действительности в первом примере мера Гиббса является мерой Бернулли в каждой из трех областей: $\nu>\lambda, \nu=\lambda, \nu<\lambda$, подробнее см. [10].

Критический случай и скейлинговый предел. Есть два способа рассмотрения скейлинг-предела. Первьй состоит в рассмотрении динамики для критических значений параметров (в первом примере это $\frac{\lambda}{\mu}=1$ ). Критические экспоненты получаются непосредственно из динамики. Сушествуют результаты для достаточно общих переходов: для данных двух положительных функций $\nu(s), \lambda\left(s, s^{\prime}\right)$ определим интенсивности перехода как

$$
\nu\left(s_{1} \ldots s_{N} \rightarrow s_{1} \ldots s_{N-1}\right)=\nu\left(s_{N}\right), \quad \lambda\left(s_{1} \ldots s_{N} \rightarrow s_{1} \ldots s_{N+1}\right)=\lambda\left(s_{N}, s_{N+1}\right),
$$

они зависят, таким образом, от крайнего правого символа. Предположим, что функции $\nu(s), \lambda\left(s, s^{\prime}\right)$ таковы, что цепь Маркова нулевая возвратная, условия для этого см. в [11]. Пусть $S=\left\{s_{1}, \ldots, s_{k}\right\}$ конечно. Пусть $n(t)=\left(n_{1}(t), \ldots, n_{k}(t)\right), n_{i}(t)-$ число символов $s_{i}$ в цепочке $\sigma(t)$. Тогда 
ТЕОрема 2. Для случайного вектора $n(t)$ имеет место чентральная предельная теорема, т.е. существует предел по вероятности при $t \rightarrow \infty$

$$
\frac{n(t)}{\sqrt{t}} \rightarrow|w| c
$$

где ш имеет стандартное гауссово распределение, а с-постоянный вектор.

Это дает те же экспоненты, что и при равновесном подходе. Заметим, что в обратимом случае доказательство сводится к случайному блужданию с отражением. Доказательство для необратимой динамики сложнее: для конечного $S$ его можно найти в [11]. Для компактного $S$ оно отсутствует, но, по-видимому, должно быть аналогичным. Было бы интересно найти примеры для некомпактного случая с негауссовым предельным распределением.

Второй подход соответствует скейлинг-пределу в равновесном случае. В динамике делается скейлинг параметров одновременно со скейлингом времени $t$, при этом разность $\nu-\lambda$ стремится к нулю и шкалируется как $t^{-\frac{1}{2}}$. Такой скейлинг-предел в динамике соответствует диффузионному приближению в массовом обслуживании. При этом при скейлинге (с постоянными $\tau, r)$

$$
t=\tau N, \quad x=r \sqrt{N}, \quad \nu-\lambda=N^{-\frac{1}{2}}, \quad N \rightarrow \infty
$$

получается броуновское движение со сносом. Снос определяет массовую щель в спектре генератора соответствующего диффузионного процесса.

Случайные грамматики. Мы рассматривали динамику, называемую линейной справа грамматикой (не обязательно контекстно свободной, см. [6]), в терминологии информатики. Теперь рассмотрим более общую динамику, в которой переходы могут быть в любом месте цепочки, а не только на ее правом конце.

Для первого примера можно построить следующую обратимую цеп Маркова, оставляющую инвариантной нашу меру, которая является контекстно-свободной случайной грамматикой (см. определение в [6]). При этом каждый символ цепочки удаляется с интенсивностью $\nu$, и для каждого $i=0,1, \ldots, n$ вставляется новьй символ между символами $s_{i}$ и $s_{i+1}$ (если $i=0$, мы ставим его перед $s_{1}$, и для $i=n-$ после $s_{n}$ ) цепочки $s_{1} \ldots s_{n}$ с интенсивностью $\lambda$. Добавляемый символ с вероятностью $\frac{1}{2 d}$ принимает одно из $2 d$ значений координатного вектора $e$. Для доказательства инвариантности меры заметим, что длины пути также образуют цеп Маркова (на $\mathbb{Z}_{+}$). Это процесс рождения-гибели на $\mathbb{Z}_{+}$с интенсивностями скачков $q_{i, i+1}=\lambda i, q_{i+1, i}=\nu i$, $q_{0,1}=\lambda$. Тогда его стационарные вероятности равны $($ если $\lambda<\nu)$

$$
\pi_{k}=\frac{q_{0,1} q_{1,2} \cdots q_{k-1, k}}{q_{1,0} \cdots q_{k, k-1}}=C \exp (-\mu k), \quad \mu=\ln \frac{\nu}{\lambda}
$$

Для двух других примеров динамику (не контекстно-свободную) также можно построить, мы рассмотрим это в другой статье в общем случае. 


\section{3. Спиновые комплексы}

3.1. Клеточные структуры. Здесь мы даем необходимый минимум основных определений касательно клеточных структур.

Комплекс строится посредством склеивания элементарных компонент - клеток, аналогично тому как материя состоит из молекул. С одной стороны, надо быть очень аккуратным при формулировке правил склеивания и возникающих вероятностных распределений. С другой стороны, кажется сомнительным, что некоторые типы клеточных структур имеют преимущества перед другими. Нет никаких физических причин предпочитать одну клеточную структуру или правило склейки другим. Таким образом, разные возможности должны быть рассмотрены, чтобы проверить универсальность некоторых законов. В этой статье мы встретим два универсальных класса, один из которых популярен в физике. Кроме того, имея гибкость в выборе клеточной структуры, можно получить более простые и даже решаемые модели.

3.1.1. Абстрактные комплексы. (Маркированным) комплексом $Г$ называется множество элементов, называемых клетками, причем определена функция $\operatorname{dim} A$ на $\Gamma$ - размерность клетки $A$, принимающая значения $0,1,2, \ldots$ Размерность $\Gamma$ есть $\operatorname{dim} \Gamma=\sup _{A} \operatorname{dim} A$. Пусть $\Gamma_{d} \subset \Gamma-$ множество клеток размерности $d$. Для каждой клетки $A \in \Gamma_{d}, d>0$, определено подмножество $\partial A \subset \bigcup_{i=0}^{d-1} \Gamma_{i}$, гранища $A$. Подкомплекс $\Gamma^{\prime}$ комплекса $\Gamma$ есть такое подмножество $\Gamma$, что если $A \in \Gamma^{\prime}$, то и $\partial A \subset \Gamma^{\prime}$.

Изоморфизм двух комплексов - взаимно однозначное отображение, сохраняюшее размерность и границы. Классы эквивалентности комплексов по отношению к изоморфизму назьваются немаркированными комплексами.

Заметим, что сами комплексы Г можно рассматривать как частный случай спиновых граффов $(G, s)$, см. [7]. Это соответствие можно осушествить разными путями. Например, пусть вершины $i$ графа $G$ соответствуют клеткам $\Gamma$, функция $s(i)$ равна размерности соответствующей клетки. Ребра определяются матрищей инцидентности: две вершины $A$ и $A^{\prime}$ графа $G$ (т.е. две клетки $Г$ ) связаны ребром тогда и только тогда, когда $A^{\prime} \in \partial A$.

(Маркированный) спиновьй комплекс это пара $(\Gamma, s)$, где $\Gamma-$ комплекс и $s: C(\Gamma) \rightarrow S$ функция на множестве $C(\Gamma)$ клеток комплекса $\Gamma$ со значениями в некотором спиновом пространстве $S$. Изоморфизм двух спиновых комплексов есть изоморфизм комплексов, сохраняющий спины. Классы эквивалентности называются (немаркированными) спиновыми комплексами. Если не оговорено противное, мы будем рассматривать только функции $s$, определенные на клетках максимальной размерности; иногда можно считать функцию $s$ определенной на вершинах двойственного комплекса, если двойственньй комплекс сушествует.

3.1.2. Топологические комплексы. Есть много топологических инкарнаций абстрактных комплексов. В каждой из них клетка представлена открытым диском. $\mathrm{CW}$-комплекс есть топологическое пространство $K$, являюшееся объединением своих $d$-мерных скелетов $K_{d}$, которые определяются по индукции следующим образом. Пусть $K_{0}=\Gamma_{0}-$ несвязное объединение точек (вершин) - клеток размерности 0 . В общем случае, $K_{d}$ получается из $K_{d-1}$ присоединением некоторого числа клеток $A$ размерности $d$ следующим образом. Каждая такая клетка $A$ размерности $d$ отождествляется с открытым $d$-мерным диском $D_{A}$, и при этом выбирается некоторое 
непрерьвное (приклеивающее) отображение $\phi_{A}: \partial D_{A} \rightarrow K_{d-1}$. Тогда $K_{d}$ определяется как фактор-пространство пространства, полученного объединением $K_{d-1}$ с $\bigcup_{A: \operatorname{dim} A=d} D_{A}$ с дальнейшим отождествлением $x \in \partial D_{A}$ с $\phi_{A}(x)$.

Например, $K_{1}$ есть граф с вершинами - нульмерными клетками, и ребрами - одномерньми клетками. Ребро $A$ называется петлей, если граница $A$ отображается на одну вершину. Часто на приклеивающее отображение налагаются некоторые ограничения. Мы ограничиваемся здесь случаем $\operatorname{dim} \Gamma=2$, причем для всех $A \mathrm{c} \operatorname{dim} A=2$ граница $\partial D_{A}$ является объединением некоторых клеток $B, \operatorname{dim} B=0,1$ (в некоторых книгах CW-комплексы так и определяются, см. [12]). C таким CW-комплексом можно ассоциировать абстрактный комплекс $\partial A=\left\{B \in K_{0} \cup K_{1}: B \subset \phi_{A}\left(D_{A}\right)\right\}$.

Мы получаем класс симплициальных комплексов (где клетки называются симплексами), если для любой клетки $A$ множество $\partial A \cap K_{0}$ вершин однозначно определяет $A$, а граница каждой двумерной клетки содержит точно 3 одномерных клетки. В частности, заметим, что всякий граф̆ без кратных ребер и без петель является симплициальным комплексом размерности 1.

3.1.3. Клеточные поверхности. Мы рассматриваем в статье различные классы двумерных комплексов. Класс определяется некоторыми ограничениями на введенньй вьше (максимальньй) класс комплексов или конструктивной процедурой, которой можно получить все комплексы этого класса. Иначе можно сказать, что класс определяется как язык, полученньй с помощью некоторой грамматики на графах, см. [6], [7].

Следующие ограничения имеют место для всех комплексов в этой статье: комплекс является (замкнутой компактной) поверхностью. Псевдоповерхностью (замкнутой) называется топологическое пространство, изоморфное конечному 2-мерному симплициальному комплексу со следующим дополнительным свойством: каждое ребро принадлежит границе ровно двух двумерных клеток. Поверхность отличается дополнительным свойством: каждая вершина имеет открытую окрестность, гомеоморфную диску.

Вот список всех компактных замкнутых (без дырок) двумерных поверхностей. Ориентированные поверхности: $S_{\rho}, \rho=0,1,2, \ldots,-$ сферы с $\rho$ ручками. Неориентируемые поверхности: $P_{1}$ (проективная плоскость), $P_{2}$ (бутылка Клейна) $, P_{k}, k=3, \ldots,-$ сфера, в которой вырезано $k$ дырок и каждая из них заклеена лентой Мёбиуса (crosscup) так, что гранища ленты Мёбиуса отождествлена с границей дырки.

Если комплекс изоморфен такому пространству, то $K_{1}$ является графом, гомеоморфно вложенным в поверхность $S$. Такие комплексы изучаются в топологической теории граффов (см. [13]) и в комбинаторике, где они называются картами. Поверхность с дырками получается из замкнутой поверхности вырезанием конечного числа дисков с непересекающимися границами. Гранища поверхности с дырками принадлежит $K_{1}$.

Изоморфизм карт есть изоморфизм абстрактных комплексов. Другими словами, две карты изоморфны, если есть гомеоморфизм $S$, отображающий вершины на вершины, ребра на ребра и грани (двумерные клетки) на грани. Мы здесь будем рассматривать только отображения, сохраняюшие ориентацию.

Карта $B$ есть подразбиение карты $A$, если граф $K_{1}(A)$ карты $A$ является подграфом графа $K_{1}(B)$ карты $B$. Известно, что два двумерных (и даже трехмерных) топологических комплекса гомеоморфны как топологические пространства, если существуют 
их подразбиения, изоморфные как абстрактные комплексы.

Эйлерова характеристика комплекса (для компактной замкнутой поверхности) определяется как

$$
\chi=V-L+F
$$

где $F$ - число граней, $V$ - число вершпн, $L$ - число ребер. Она не зависит от вида комплекса, а только от самой поверхности: для ориентируемых поверхностей $\chi=2-2 \rho$, где $\rho$ - род (число ручек), для неориентируемых $\chi=2-n$, где $n$ - число листов Мёбиуса (crosscups).

В дальнейшем нас будут интересовать в основном следующие 5 классов комплексов (карт).

Произвольные карты. Это тот класс, которьй мы только что определили. Никаких дополнительных ограничений не накладывается. Простейшие примеры: одна вершина внутри сферы - карта-вершина, ребро с двумя вершпнами внутри сферы карта-ребро.

Гладкие клеточные поверхности. Гладкая клеточная поверхность (см. [14]) есть компактное связное двумерное многообразие $M$ с конечным числом замкнутых подмножеств (клеток) $F_{i}$ таких, что:

1) $\bigcup F_{i}=M$;

2) для каждого $i$ сушествует гладкое однозначное отображение $f_{i}$ клетки $F_{i}$ на полигон с $n_{i} \geqslant 3$ ребрами;

3) если $i \neq j$, то либо $F_{i} \cap F_{j}=\varnothing$, либо $f_{i}\left(F_{i} \cap F_{j}\right)$ является ребром или вершиной соответствующего полигона.

Триангуляции. Это гладкие клеточные поверхности, где все $n_{i}=3$.

Множество $V$ вершин некоторого комплекса называется разделяюшим, если есть два подграффа $G_{1}, G_{2}$ таких, что $G_{1} \cup G_{2}=G, G_{1} \cap G_{2}=V$. Для триангуляций отсутствие разделяюших множеств эквивалентно отсутствию петель у $K_{1}$.

Триангуляция диска определяется как гладкая клеточная поверхность, гомеоморфная диску. При этом нет разделяюших множеств из одной точки. Тогда ее можно рассматривать как триангуляцию сферы, в которой одна грань (соответствующая внешности диска и называемая внешней гранью) выделена, а для всех остальных граней $n_{i}=3$.

Симплициальные комплексы. Это триангулящии без кратных ребер, где, кроме того, три произвольных вершины триангулящии определяют не более одной клетки. Заметим, что треугольник (цикл длины 3), содержаший внутри и вне по крайней мере по одной вершине, не определяет клетки.

Выпуклые полиэдры. Вьпуклые полиэдры есть частньй случай карт с $\rho=0$. Существует чисто комбинаторная характеризация карт, соответствующих выпуклым полиэдрам. Если триангуляция не имеет ни петель, ни кратных ребер, то, если $L \geqslant 4$, она соответствует (по теореме Штейница-Радемахера) выпуклому полиэдру.

3.1.4. Локальный наблюдатель (корень). Комплекс назьвается помеченным, если множество вершин занумеровано. Здесь есть один важньй нюанс: часто не говорят о нумерации, подразумевая, что множество вершин задано некоторым образом. Однако как метки, так и фиксация множества вершин полезны для фиксации координатной системы, но являются лишпими для геометрии и топологии. В то же время классы эквивалентности комплексов неудобны по другим причинам. Есть другой 
способ избежать лишней координатизации, имея в то же время тривиальную групу автоморфизмов. Это по сушеству та же самая координатизация, с помошњю которой можно по некоторому алгоритму занумеровать вершины комплекса. Корень (или локальный наблюдатель) в помеченном комплексе есть набор $(f, l, v)$, где $f$ - двумерная клетка, $l$ - одно из ее ребер, $v$ - одна из вершин $l$. Изоморфизм двух комплексов с корнями есть изоморфизм комплексов, уважающий корни, но не сохраняющий нумерацию. Корневая карта (корневой комплекс, комплекс с локальным наблюдателем) класса А есть класс эквивалентности комплексов с корнем в классе А. Мы будем всегда предполагать, что корневое ребро ориентировано и направлено от корневой вершины. Для триангуляций диска корневой будет всегда внешняя грань $i$, при этом, возможно, $n_{i} \neq 3$.

Лемма 2. Группа автоморфизмов любой корневой карты тривиальна.

Это легко доказывается индукцией по числу клеток, если распространять автоморфизм последовательно с корневой клетки на смежные.

3.1.5. Движения. Грамматики на графрах, соответствуюшие преобразованиям комплексов, изучены мало. Соответствующие подстановки назьваются здесь движениями. В следуюшем разделе 4 мы рассмотрим движения Тутте, см. рис. 5, которые состоят в добавлении ребра между двумя вершинами клетки или объединении двух комплексов отождествлением двух их вершин. В топологии, однако, всегда большую роль играли разбиения. В двух статьях [15], [16] некоторые движения детально изучены.

Пусть $A$ - коммутативная ассоциативная алгебра над $\mathbb{Z}_{2}$ (симплициальные цепи над $\mathbb{Z}_{2}$ ), порожденная символами из некоторого счетного алфавита $L$ с коммутационными соотношениями $s_{i}^{2}=0, s_{i} s_{j}=s_{j} s_{i}$. Таким образом, это линейная оболочка, порожденная цепочками (симплексами) $\alpha=s_{1} \ldots s_{n}$. Определим граничный оператор как линейный оператор $\partial: A \rightarrow A$ такой, что

$$
\partial \alpha=\sum \beta
$$

где сумма берется по всем подмономам $\beta=s_{i_{1}} \ldots s_{i_{n-1}}, 1 \leqslant i_{1}<\cdots<i_{n-1} \leqslant n$, монома $\alpha$. Мы будем здесь рассматривать только двумерные цепи, т.е. $n \leqslant 2$.

Другие линейные операторы в этой алгебре назьваются движениями Александера $S_{i, j ; x}, i, j, x \in L$. Они определяются следуюшим образом:

$$
S_{i, j ; x} \mathrm{a}=x(i+j) \beta,
$$

если $\alpha=i j \beta$, и равно 0 в остальных случаях.

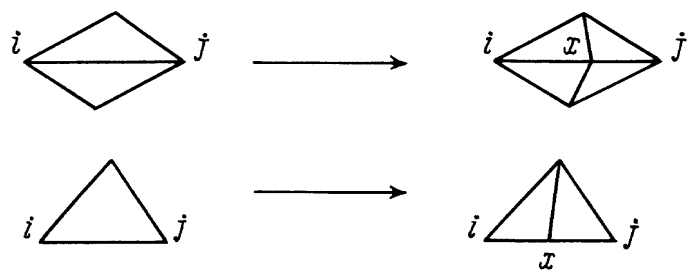

Рис. 2. Движения Александера 


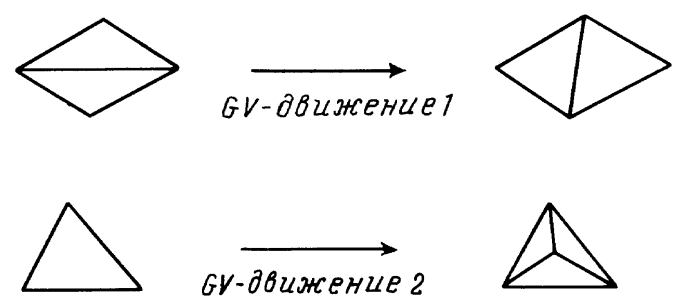

Рис. 3. Движения Гросса-Варстеда

Следуюший пример - движения Гросса-Варстеда, определенные на рис. 3. Можно доказать, см. [16], что каждое движение Александера является некоторой композицией движений Гросса-Варстеда и наоборот. Мы скажем, что множество движений $R$ неприводимо в классе $\mathbf{A}$ комплексов, если для любой пары $T_{1}, T_{2}$ комплексов из $\mathbf{A}$ существует последовательность движений из $R$, дающих $T_{2}$ из $T_{1}$ (в физической литературе в этом случае используется термин "эргодическое", но мы хотим пользоваться стандартной вероятностной терминологией).

Tеорема 3. В классе А симплициальных комплексов множество движений Александера и множсество движений Гросса-Варстеда неприводимы.

Доказательство см. в [15], [16].

3.1.6. Группа автоморфизмов. Пусть $\mathbf{A}$ - один из классов комплексов, введенных выше.

Теорема 4. Для почти всех комплексов с $N$ гранями (т.е. двумерными клетками) из $\mathbf{A}$ группа автоморфизмов тривиальна, т.е. $\frac{\mathbf{A}_{\text {nontrivial }(N)}}{\mathbf{A}(N)} \rightarrow 0$ при $N \rightarrow \infty$, әде $\mathbf{A}(N)\left(\mathbf{A}_{\text {nontrivial }}(N)\right)$ - множество всех комплексов с $N$ гранями из $\mathbf{A}$ (то же, но с нетривиальной группой автоморфизмов).

Ранее Тутте заметил, что это утверждение интуитивно ясно. Много строгих результатов появилось с тех пор, см. [17]-[19]. Доказательство для триангуляций диска см. в [20].

3.2. Метрика и кривизна. Метрика на комплексе определена, если она определена на каждой замкнутой клетке так, что метрики на границах клеток совпадают. Существует два основных подхода к определению метрики: динамические триангуляции - когда все ребра имеют единичную длину, и квантовое исчисление Редже - когда они случайны. Мы примем первый подход. В нем клетки с одинаковым числом ребер идентичны и метрика на них стандартна.

Можно поступить по-другому: сначала рассмотрим граф $K_{1}$, вложенньй в плоскость в виде системы гладких дуг. Определим сначала метрику на графе $K_{1}$ так, чтобы все длины ребер равнялись константе, а плошадь граней (если это треугольники) равнялась 1 . Внутри клетки с $n$ ребрами определим метрику посредством гладкого взаимно однозначного отображения равностороннего полигона $Q_{n}$ с $n$ ребрами на эту клетку так, чтобы гладкость имела место в окрестности ребер. Тогда внутри граней кривизна равна нулю. То же самое на гранях вне вершин, это показано на рисунке в кусочно-линейном случае. 

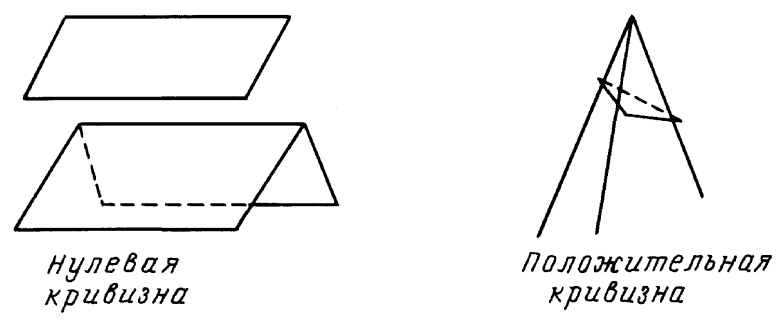

Рис. 4. Кривизна

Определим кривизну $R_{v}$ в вершине $v$. Как всегда, кривизна измеряется параллельным переносом (связность Леви-Чивита) вектора (лежащего в плоскости грани в кусочно-линейном случае) вдоль замкнутого пути: вдоль внутренней части грани - как на плоскости, через ребро - разгибая две сложенные полуплоскости. Таким образом, только пути вокруг вершин могут дать ненулевую кривизну. Вокруг вершины $v$ угол между начальным и перенесенным векторами равен $\varepsilon_{v}=2 \pi-\sum_{f} \varphi_{f v}$, где $\varphi_{f v}-$ угол симплекса $f$ в вершине $v$. Заметим, что

$$
2 \pi V-\sum_{v} \varepsilon_{v}=\sum_{v} \sum_{f} \varphi_{f v}=\sum_{f} \sum_{v} \varphi_{f v}=\pi F .
$$

Отсюда и из формулы Эйлера $\chi=V-L+F$ следует формула Гаусса-Боннэ

$$
\sum_{v} \varepsilon_{v}=2 \pi \chi
$$

для триангуляций, так как $L=\frac{3}{2} F$.

Классические примеры: положительная кривизна - эллиптическая геометрия (сфера, проективная плоскость); нулевая кривизна - евклидова геометрия (плоскость, тор, бутылка Клейна); отрицательная кривизна - гиперболическая геометрия (все остальные).

Сейчас мы покажем, как кривизна $R_{i}$ в вершине $i$ определяется числом $q_{i}$ ребер, инцидентных $i$. Действие Эйнштейна-Гильберта на гладком многообразии равно

$$
\int\left(c_{1} R+c_{2}\right) \sqrt{g} d x
$$

где $R$ - гауссова кривизна, $g$ - метрика. По формуле Гаусса-Боннэ для гладких поверхностей $\int R d \sigma=\int R \sqrt{g} d x=4 \pi \chi$. Ее связь с дискретным случаем для разбиения сферы на полигоны с $n_{i}$ ребрами и с площадьми $A_{i}$ (площадь $A$ треугольника равна $\left.A=\alpha_{1}+\alpha_{2}+\alpha_{3}-\pi\right)$ дается формулой

$\sum k_{i} A_{i}=\sum_{i}\left[\sum_{j} \alpha_{i j}-\left(n_{i}-2\right) \pi\right]=\sum_{i j} \alpha_{i j}-\pi \sum n_{i}+2 \pi F=2 \pi V-2 \pi E+2 \pi F$,

где $\alpha_{i j}$ - углы клетки. Таким образом, дискретное действие должно быть равно (с точностью до константы) $\lambda \rho+\mu N$, где $\rho$ - род и $N$ - число треугольников. Мы, 
однако, хотим написать это действие через кривизну, суммируя по вершинам вместо суммирования по треугольникам. Все треугольники равносторонние, и пусть их площадь равна 1. Тогда каждая вершина получает площадь $\frac{1}{3}$ от каждого инцидентного треугольника, т.е. $\frac{q_{i}}{3}$ в обшей сложности. Поэтому

$$
\int R d \sigma \simeq \sum_{i} \frac{q_{i}}{3} R_{i}
$$

и формула

$$
\sum_{i} \frac{q_{i}}{3} R_{i}=4 \pi \chi
$$

имеет место, только если положить $R_{i}=2 \pi \frac{6-q_{i}}{q_{i}}$.

\section{4. Равновесная чистая плоская гравитация}

Два вида техники используются в двумерной квантовой гравитации. Исторически первая из них - комбинаторный подход, начатый Тутте и продолженный (без всякого упоминания о физике) многими исследователями, их статьи публикуются в комбинаторных журналах. Второй - случайные матричные модели (CMM), начатый в самой физике. Вычисления во втором подходе весьма убедительны, но не до конща строги. Насколько известно автору, никаких математически строгих связей между двумя подходами не установлено. Мы используем первый подход и дадим краткий обзор второго.

4.1. Определения и комбинаторный подход. Пусть $\mathbf{A}$ - некоторый класс комплексов $T$ (например, определенный в предыдушем разделе), гомеоморфных сфере, $|T|=F(T)$ - число клеток размерности 2 в $T, C(N)=\downarrow\{T \in \mathbf{A}:|T|=N\}$. Основный пример будет класс всех триангулящий сферы.

Большой канонический ансамбль определяется как

(2) $P(T)=Z^{-1} \exp (-\mu F(T)), \quad Z=\sum_{T \in \mathbf{A}} \exp (-\mu F(T))=\sum_{N} C(N) \exp (-\mu N)$.

В частности, условное распределение $T \in \mathbf{A}$ с фиксированным $N$ равномерно. Есть простой, общий и часто полезньй метод грубой оценки $C(N)$.

ЛЕмма 3 (априорные экспоненциальные оценки). $c_{1} \gamma_{l}^{N}<C(N)<c_{2} \gamma_{u}^{N}, 1<\gamma_{l}<$ $\gamma_{u}<\infty, c_{i}>0$

ДокАЗАТЕльство. Идея доказательства оценки снизу состоит в том, что сфера, разбитая на $N$ треугольников, представляется вытянутой в трубку длины порядка $N$ и поперечного сечения порядка константы $n$ (метрика здесь определяется тем, что длины всех ребер равны 1). Возьмем два неизоморфных комплекса, гомеоморфных кольцу на плоскости, с одинаковым числом ребер с обеих сторон и имеющих $n$ клеток. Первьй комплекс: чередуюшиеся через один треугольники, стояшиеся на ребре и на вершине; второй - два на ребре, два на вершине и т. д. Склеим их последовательно один за другим в произвольном порядке. Так мы получаем нижнюю оценку $2^{\frac{N}{n}}$. 
Приводимый ниже метод доказательства верхней оценки работает даже в более общей ситуации. Можно предложить алгоритм построения всех возможных комплексов с $N$ клетками размерности 2 . Начнем с одной клетки. Занумеруем ее ребра как 1, 2, 3 . На каждом шаге будем добавлять не более одной клетки к границе и будем нумеровать новые ребра в порядке их появления. Опишем эту индуктивную конструкцию. Возьмем ребро с номером один и примем одно из 4 решений: 1) не добавлять более треугольников к данному ребру, 2) добавить к нему клетку с точно двумя новыми ребрами, 3) добавить клетку к этому ребру и к соседнему (в направлении по часовой стрелке), т.е. с одним новым ребром, 4) то же самое в направлении против часовой стрелки. Для каждой из $4^{k}$ последовательностей решений $\omega$ пусть $f(k, \omega)$ - число ребер после $k$ шагов, $f(k, \omega) \leqslant 3+2 k$, так как на каждом шаге добавляется не более двух ребер. Кроме того, если есть $n$ клеток, то не может быть более $3 n$ решений типа 1.

Однако необходима точная асимптотика. Все известные примеры показывают следующее асимптотическое поведение:

$$
C(N) \sim c_{1} N^{\alpha} c^{N}
$$

Из (3) вытекает

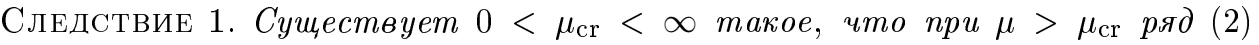
сходится. Он расходится, если $\mu<\mu_{\mathrm{cr}}$. Если $\mu=\mu_{\mathrm{cr}}$, то $Z<\infty$, если и только если $\alpha<-1$.

Таким образом, при $\mu<\mu_{\mathrm{cr}}$ распределения не существует. Однако, динамика, вводимая ниже, позволяет дать интерпретацию и таким $\mu$.

Ни одна из констант $c_{1}>0, c>1$ не универсальна, но для всех известных примеров $\alpha$ универсальна (имеет одно и то же значение для всех примеров). Универсальность $\alpha$ вовсе не такой уж интуитивньй факт. Например, предсказания, основанные на нестрогих физических аргументах (фактически на методе перевала, см., например, [21]), не дали правильного численного значения $\alpha=-\frac{7}{2}$ даже в планарном случае.

ТЕОРема 5. Асимптотика (3) имеет место для всех пяти классов, определенных в разделе 3.1.3. Более того, $\alpha=-\frac{7}{2}$ во всех этих случаях.

ДокАЗАТЕЛЬСтво. Мы докажем это только для триангуляций; остальные случаи рассеяны по ссылкам [17]-[20], [22]-[26]. Подобным образом определим распределение на классе $\mathbf{A}_{0}$ корневых комплексов (триангуляции с выделенным “корнем"):

$$
P_{0}(T)=Z_{0}^{-1} \exp (-\mu F(T)), \quad Z_{0}=\sum_{T \in \mathbf{A}_{0}} \exp (-\mu F(T))=\sum_{N} C_{0}(N) \exp (-\mu N)
$$

где индекс 0 означает, что рассматриваются только корневые комплексы класса $\mathbf{A}$.

ЛЕМма 4. Для триангуляций

$$
C(N) \sim(3 N)^{-1} C_{0}(N)
$$

Это следует из тривиальности группы автоморфизмов для большинства комплексов. Действительно, некорневой комплекс можно сделать корневым, выделив сначала клетку размерности 2 , что дает множитель $N$, если группа автоморфизмов комплекса 
тривиальна. Аналогично, выбор одного из трех ребер выделенной клетки дает множитель 3.

Обозначим $C(N, m)$ число триангулящий диска, где внешняя грань (содержашая северный полюс сферы) имеет $m$ ребер, $C_{0}(N, m)$ - число таковых, где, кроме того, внешняя грань корневая (иначе говоря, одно из ребер границы выделено и ориентировано против часовой стрелки). Следующий результат подобен предыдущему, но доказьвается проше.

Лемма 5. $C(N, m) \sim m^{-1} C_{0}(N, m)$ для больиих $N$ и фиксированного $m$.

ДокАЗАтЕЛЬство. Рассмотрим теперь автоморфизмы диска. Перенумеруем в циклическом порядке ребра границы: $1,2, \ldots, m$. Автоморфизм диска $\phi$ однозначно определен, если задано $j=\phi(1)$, т.е. если задано ребро, в которое переходит ребро с номером 1. Действительно, тогда примыкаюший к ребру 1 треугольник переходит в треугольник, примыкаюший к $j$, и так далее по связности.

Мы покажем, что для заданного $j$ сушествует гораздо больше (для больших $N$ ) комплексов таких, что у них нет автоморфизма $\phi$ такого, что $j=\phi(1)$. Покажем, что каждый комплекс $A$, имеюший нетождественный автоморфизм $\phi$, может быть разбит на два подкомплекса $A_{1} \cup A_{2}=A$ так, что каждая грань принадлежит только одному из них и $\phi A_{1}=A_{2}$. Это делается по индукции следующим образом. Возьмем некоторое граничное ребро, возьмем треугольник $T$ с этим ребром и отнесем его к $A_{1}$, положим тогда $\phi T \in A_{2}$. Каждьй шаг индукции состоит из взятия еще одного треугольника, имеющего обшее ребро с уже построенной частью $A_{1}$. После того можно изменить $A_{2}$ внутри некоторым числом $u(N)$ способов, ограниченньм снизу некоторой функцией $u(N) \rightarrow \infty, N \rightarrow \infty$, равномерно по $A$. Это можно сделать так: выберем $u(N)$ треугольников в $A_{2}$, не слишком близко друг от друга, и изменим независимо окрестность каждого из них, оставляя границу окрестности и число клеток $n$ в этой окрестности неизменными. Это возможно, так как $C(n, b)>1$, где $b$ - число ребер на границе. Таким образом, при данном $A_{1}$ пропорция комплексов с $\phi A_{1}=A_{2}$ мала. Мы доказали, что очень мало комплексов имеют автоморфизм $\phi$ такой, что $j=\phi(1)$. Так как $m$ фиксировано, то это верно для всех $j$.

Ввиду вьшедоказанных лемм для доказательства теоремы остается доказать, что $C_{0}(N) \sim c_{2} N^{-\frac{5}{2}} c^{N}$

Универсальньй характер формулы (3) сильно подкрепляется фактом (доказьваемьм ниже для триангулящий), что для всех таких примеров первая положительная сингулярность производящей функции $\sum_{N} C_{0}(N) z^{N}$ является алгебраической сингулярностью, что и дает (3).

ЗАмЕчАниЕ 1. Предположим, что алгебраическая функция $y(x)$ аналитична в точке 0 и имеет минимальную положительную сингулярность в точке $a>0$. Скажем, что ведущая экспонента равна $b=\max b_{i}, i=1,2, \ldots, d$, где $b_{i} \neq 0,1,2, \ldots$ рациональны, если существуют такие $b_{i}$ и функции $g_{i}(x), i=0,1, \ldots, d$, аналитические в $x=a$ и такие, что

$$
y(x)=\sum_{i=1}^{n} g_{i}(x)\left(1-\frac{x}{a}\right)^{b_{i}}+g_{0}(x) .
$$


Тогда мы имеем разложение (это утверждение известно как теорема Дарбу)

$$
y(x)=\sum_{n} c_{n} x^{n}, \quad c_{n} \sim \frac{g_{1}(a)}{\Gamma(-b)} n^{-b-1} a^{-n} .
$$

В нашем случае (для $\left.C_{0}(N)\right) b=\frac{3}{2}$. Можно применять также тауберовы теоремы в подобной ситуации.

Дадим несколько примеров, где все константы в асимптотике известны, см. [22] и библиограффию там же. Первый пример - класс всех триангуляций. Здесь $C_{0}(N) \sim$ $\gamma_{2} N^{-\frac{5}{2}} c^{N}, c=3 \sqrt{\frac{3}{2}}$. Для вьпуклых полиэдров $C_{0}(N) \sim \gamma_{3} N^{-\frac{5}{2}} c_{1}^{N}, c_{1}=\frac{16}{3 \sqrt{3}}$. Для симплициальных триангулящий $C_{0}(N) \sim \gamma_{4} N^{-\frac{5}{2}} c^{N}, c=\frac{3 \sqrt{3}}{2}$. Можно дать много других примеров; однако интереснее понять общий механизм, на котором это держится.

Тутте [23]-[25] начал исследовать асимптотику $C(N, m)$ и развил мощньй "квадратичный" метод. Впоследствии многие авторы развили этот метод и получили асимптотику для различных классов $\mathbf{A}$ (см. обзор [26] и более позднюю работу [20]).

Главная идея Тутте - следуюшие рекуррентные уравнения для $C(N, m), N=$ $0,1, \ldots ; m=2,3, \ldots$ :

$$
\begin{gathered}
C(N, m)=C(N-1, m+1)+\sum_{\substack{N_{1}+N_{2}=N-1, m_{1}+m_{2}=m+1}} C\left(N_{1}, m_{1}\right) C\left(N_{2}, m_{2}\right), \quad m \geqslant 3, N \geqslant 1, \\
C(0,2)=1, \quad C(0, m)=0, \quad m>2 .
\end{gathered}
$$

Эти уравнения могут быть получены из картинки, см. рис. 5, где ориентация корневого ребра отмечена стрелкой, корневая грань (содержашая северньй полюс сферы) справа от стрелки. Эти картинки определяют два типа движений, называемых движениями Тутте. Рассмотрим произвольную корневую карту с параметрами $(N-1, m+1)$ и сделаем движение Тутте типа 1 (линейное), возьмем упорядоченную пару корневых карт $\left(N_{1}, m_{1}\right),\left(N_{2}, m_{2}\right)$ и вьполним движение Тутте типа 2 . Так может быть получена любая корневая карта $(N, m)$. $(0,2)$ соответствует так называемому комплексу-ребру с одним ребром, которое считается дважды.

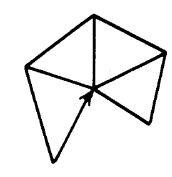

$$
\begin{aligned}
& \text { Линецное } \\
& \text { движение }
\end{aligned}
$$
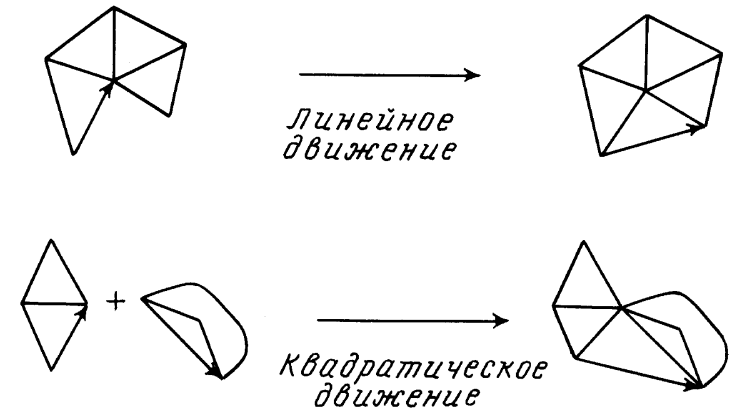

Рис. 5. Линейные и квадратические движения 
Если ввести производящую функцию

$$
U(x, y)=\sum_{N=0}^{\infty} \sum_{m=2}^{\infty} C(N, m) x^{N} y^{m-2}
$$

то, умножая обе стороны рекуррентного уравнения на $x^{N} y^{m}$ и складывая, получим функциональное уравнение

$$
U(x, y)=U(x, y) x y^{-1}+U^{2}(x, y) x y+1-x y^{-1} U(x, 0) .
$$

Ниже мы выведем отсюда, что $U(x, 0)$ - алгебраическая функция, и вычислим ее ведущую сингулярность в немного более общей ситуации.

Функции Грина. Рассмотрим некоторый класс А комплексов. Пусть $\mathbf{A}\left(m_{1}, \ldots, m_{k}\right)$ - класс комплексов, определенных с теми же ограничениями, что и $\mathbf{A}$, гомеоморфных сфере с $k$ дырками и с $m_{i}$ ребрами на границах этих дырок. Предположим, что эти границы не пересекаются. Функции Грина определяются следующим образом:

$$
Z\left(m_{1}, \ldots, m_{k}\right)=\sum_{T \in \mathbf{A}\left(m_{1}, \ldots, m_{k}\right)} \exp (-\mu F(T))=\sum_{N} C\left(N, m_{1}, \ldots, m_{k}\right) \exp (-\mu N)
$$

$Z$ соответствует случаю $k=0$. Корневые функции Грина определяются аналогично:

$$
Z_{0}\left(m_{1}, \ldots, m_{k}\right)=\sum_{T \in \mathbf{A}_{0}\left(m_{1}, \ldots, m_{k}\right)} \exp (-\mu F(T))=\sum_{N} C_{0}\left(N, m_{1}, \ldots, m_{k}\right) \exp (-\mu N)
$$

где индекс 0 означает, что рассматриваются комплексы с выделенным ребром на первой гранище с $m_{1}$ ребрами - локальньй наблюдатель в терминологии [6], [7]. Хотелось бы иметь выражение для функций Грина в терминах основного распределения (как это имеет место для цепей Маркова).

Функции Грина связаны с производными $\frac{d^{n} Z}{d \mu^{n}}=-\chi^{(n)}(\mu)$, т.е. с факториальными моментами $N$.

Лемма 6. Статистическая сумма и две ее первые производные конечны при $\mu=\mu_{\mathrm{cr}}$, а для $n>2$ при $\mu \rightarrow \mu_{\mathrm{cr}}+0$ имеет место соотношение

$$
\frac{d^{n} Z(\mu)}{d \mu^{n}} \sim c(n)\left(\mu-\mu_{\mathrm{cr}}\right)^{-\alpha-1-n}
$$

ДокАЗАТЕЛЬСТво. Мы увидим ниже, что $Z$ - алгебраическая функция от $z=e^{-\mu}$ и имеет главную сингулярность $C\left(1-\frac{z}{z_{0}}\right)^{-\alpha-1}$ в точке $z_{0}=e^{-\mu_{\mathrm{cr}}}$. В окрестности $z_{0}$ имеет место

$$
1-\frac{z}{z_{0}}=1-e^{-\left(\mu-\mu_{\mathrm{cr})}\right.} \sim \mu-\mu_{\mathrm{cr}}
$$

Это находится в хорошем согласии со следуюшим простым интуитивным рассуждением. 
ЛЕмма 7. Для фиксированньх $k, m_{1}, \ldots, m_{k}$ существуют константы $0<c_{1}<$ $c_{2}<\infty$ maкuе, что

$$
c_{1} N^{k-1} C_{0}(N)<C_{0}\left(N, m_{1}, \ldots, m_{k}\right)<c_{2} N^{k-1} C_{0}(N) .
$$

ДокАЗАТЕЛЬСТво. Возьмем сначала $k=1$ и докажем верхнюю оценку. Возьмем некоторый комплекс $A \in \mathbf{A}_{0}(m)$ с $N$ гранями и заклеим дырку некоторым комплексом $B \in \mathbf{A}_{0}(m)$ с $r$ гранями, где $r$ зависит только от $m$. Мы получим некоторьй комплекс $C=C(A) \in \mathbf{A}_{0}$ с $N+r$ гранями, причем таким образом можно получить не более $C_{0}(r, m)$ таких комплексов. Поэтому $C_{0}(N, m)<C_{0}(r, m) C_{0}(N+r)<c_{2} C_{0}(N)$. Нижняя оценка и индукция по $k$ проводятся аналогично. Удобнее однако, делать иначе: взять комплекс из $\mathbf{A}_{0}$ и строить из него комплексы с $k$ дырками. Для этого надо выбрать в нем случайно $k-1$ граней (что даст множитель $N^{k-1}$ ), прилегаюших к границам с $m_{2}, \ldots, m_{k}$ гранями соответственно. Далее надо провести произвольно замкнутые пути длин $m_{2}, \ldots, m_{k}$ соответственно от каждой из выбранных граней.

4.1.1. Равномерная асимптотика. Возникает два вопроса: какова асимптотика $C(N, m)$, если и $N$, и $m$ стремится к бесконечности, и какова асимптотика других глобальных переменных таких, как число вершин и т. д.? Эти два вопроса связаны.

$V, L, F$ являются случайными величинами в большом каноническом ансамбле, и можно говорить об их совместном распределении. В обшем случае только две из них независимы ввиду формулы Эйлера $V-L+F=2$. Для триангуляций, где каждая грань имеет 3 инцидентных ребра, только одна из них независима ввиду $L=\frac{3}{2} F$. Для класса всех корневых карт, где есть две независимых переменных, имеет место лемма.

ЛЕмма 8. Пусть $\mathrm{E}(V \mid F=N)$ - условное среднее число вериин, если число граней равно N. Тогда

$$
\mathrm{E}(V \mid F=N) \sim c N
$$

для некоторого $c>0$.

Как следует из формулы на с. 157 [22], число корневых карт с $N+1$ гранями и $m+1$ вершинами равно

$$
c(N, m)=\frac{1}{2 N-1} C_{2 N+m-2}^{m} \frac{1}{2 m-1} C_{2 m+N-2}^{N} .
$$

Тогда

$$
\mathrm{E}(V \mid N)=\frac{\sum_{m} m c(N, m)}{\sum_{m} c(N, m)} \sim \alpha_{\max } N,
$$

где $\alpha_{\max }$ определяется максимумом по $\alpha$ функции $c(N, m=\alpha N)$, что связано с асимптотикой больших уклонений.

Рассмотрим теперь одночастичные функции Грина.

Лемма 9. Ряд

$$
Z(\nu, \mu)=\sum_{N, m} e^{-\mu N-\nu m} C(N, m)
$$

сходится при $\nu>\nu(\mu)$, где $\nu(\mu)$ - некоторая неубьвающая функиия, возможно равная бесконечности в окрестности нуля, см. рис. 6. 


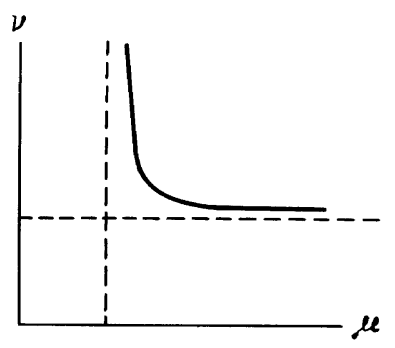

Рис. 6. Критическая кривая

ДокАЗАТЕЛЬСТво очевидно, так как ряд имеет положительные коэффициенты.

Для семейства распределений, определяемого статистической суммой $Z(\nu, \mu)$, интересно изучить асимптотику и экспоненты, когда $\mu=a \nu$, где $0 \leqslant a \leqslant \infty$ фиксироваHO.

Это легко можно сделать с помощью явной формулы (см. [22]) для числа $C_{0}(N, m)$ триангуляций с выделенным ребром на границе (корневых триангуляций)

$$
C_{0}(N, m)=\frac{2^{j+2}(2 m+3 j-1) !(2 m-3) !}{(j+1) !(2 m+2 j) !((m-2) !)^{2}},
$$

где $N=m+2 j$ - число граней помимо внешней. $C_{0}(N, m)=0$, если $N-m$ нечетно.

Тогда при $j, m \rightarrow \infty$

$$
C_{0}(N, m) \sim \operatorname{const} \frac{\sqrt{m}}{j(2 m+3 j)} 2^{j+2 m} C_{2 m+3 j}^{j},
$$

в частности, при $j \sim \beta m, \beta>0$

$$
C_{0}(N, m) \sim \operatorname{const} \frac{1}{m^{2}} 2^{j+2 m-\beta m \log \frac{\beta}{2+3 \beta}-(2+2 \beta) m \log \frac{2+2 \beta}{2+3 \beta}} .
$$

Таким образом, для всех $0<\beta<\infty$ экспонента $\alpha=-2$. Для фиксированного $m$ экспонента не зависит от $m$ и равна $\alpha=-\frac{5}{2}$ и, более того,

$$
C_{0}(N, m) \sim \phi(m) N^{-\frac{5}{2}} c^{N}
$$

Кроме того, $\phi(m) \sim C m^{\frac{1}{2}} c_{1}^{m}$ при $m \rightarrow \infty$.

4.2. Матричный подход. Модель случайных матрищ есть вероятностное распределение $\mu$ на множестве самосопряженных $(n \times n)$-матриц $\phi=\left(\phi_{i j}\right)$ с плотностью

$$
\frac{d \mu}{d \nu}=Z^{-1} \exp \left(-\operatorname{tr}\left(\frac{\phi^{2}}{2 h}\right)-\operatorname{tr}(V)\right),
$$

где $V=\sum a_{k} \phi^{k}$ - полином от $\phi$, ограниченньй снизу, $\nu$ - мера Лебега на вещественном $n^{2}$-мерном пространстве векторов $\left(\phi_{i i}, \operatorname{Re} \phi_{i j}, \operatorname{Im} \phi_{i j}, i<j\right)$. Можно это распределение переписать в виде плотности

$$
\frac{d \mu}{d \mu_{0}}=Z_{0}^{-1} \exp (-\operatorname{tr}(V))
$$


по гауссовой мере $\mu_{0}$. Легко видеть, что ковариации меры $\mu_{0}$ равны

$$
\left\langle\phi_{i j}, \phi_{k l}^{*}\right\rangle=\left\langle\phi_{i j}, \phi_{l k}\right\rangle=h \delta_{i k} \delta_{j l}
$$

Заметим, что для самого существования вероятностной меры $\mu$ необходимо, чтобы старший коэффищиент $a_{p}$ полинома $V$ был положительным, а $p$ было четным. Для этого случая сушествует хорошо развитая теория СММ моделей, которую мы здесь не рассматриваем, см. [27].

Фундаментальная связь (идущая от Хоофта) между СММ и двумерной квантовой гравитацией дается формальньм рядом по семиинвариантам или по диаграммам (см. [28])

$$
\log Z_{0}=\sum_{k=1}^{\infty} \frac{(-1)^{k}}{k !}\langle\operatorname{tr}(V), \ldots, \operatorname{tr}(V)\rangle=\sum_{k=1}^{\infty} \frac{(-1)^{k}}{k !} \sum_{D_{k}} I\left(D_{k}\right)
$$

где $\sum_{D_{k}}$ - сумма по всем связным диаграммам с $k$ вершинами. Пусть, например, $V=a_{4} \phi^{4}$. Тогда каждая диаграмма имеет занумерованные вершины $1, \ldots, k, L=2 k$ ребер, каждая вершина имеет занумерованные отростки $1,2,3,4$, соответствуюшие множителям произведения $\phi_{i j} \phi_{j k} \phi_{k l} \phi_{l i}$. Отросток, соответствуюший, например, $\phi_{i j}$, можно представлять как двустороннюю полоску, стороны которой имеют матричные индексы $i$ и $j$ соответственно. Договоримся, что спаривание отростков-полосок делается так, что спаренные стороны имеют одинаковые индексы. Так как индекс встречается дважды в каждой вершине, то, начиная с некоторой стороны с индексом $i$, есть единственный связный путь в диаграмме по ребрам с индексом $i$. Эти пути замкнуты и называются индексными петлями. Суммируя тогда по индексам, мы получим множитель $n^{N}$, где $N=N\left(D_{k}\right)$ - число индексных петель. В результате мы получим

$$
\sum \frac{\left(-a_{4}\right)^{k}}{k !} \sum_{D_{k}} h^{2 k} n^{N\left(D_{k}\right)}
$$

Для каждого графа $D$ сушествует минимальное клеточное вложение $f(D)$ этого графа в компактную ориентируемую поверхность $S_{\rho}$ рода $\rho$ (см. [13]). Клеточность вложения означает, что каждая индексная петля ограничивает открытую область поверхности $S_{\rho}$, гомеоморфную диску, а минимальность означает, что нет меньших $\rho$ с таким же свойством. Эта карта имеет $k$ вершин, $2 k$ ребер и $N$ граней. Положим $a=-a_{4}$, тогда по формуле Эйлера $k=N+2 \rho-2$ имеем

$$
\left(-a_{4}\right)^{k} h^{L\left(D_{k}\right)} n^{N\left(D_{k}\right)}=a^{k} h^{2 k} n^{N}=\left(a h^{2} n\right)^{N}\left(a h^{2}\right)^{2 \rho-2}=\left(h a^{2}\right)^{-1} \exp (-\mu N-\nu \rho)
$$

с $\mu=-\ln \left(a h^{2} n\right), \nu=-2 \ln \left(a h^{2}\right)$.

Вычисления в СММ обычно делаются только при $n \rightarrow \infty$, поэтому для получения конечных $\mu$ надо делать скейлинг $a h^{2}=b / n, b=e^{-\mu}$. В пределе $n \rightarrow \infty$ будет $\nu \rightarrow \infty$ и только члены с $\rho=0$ имеют минимальньй порядок. Предел $\lim _{\mu \rightarrow \mu_{\mathrm{cr}}} \lim _{n \rightarrow \infty}$ называется простым скейлинговьм пределом. В [29] было подсчитано, что экспонента в этом случае равна $-\frac{7}{2}$, что подтверждает универсальность экспоненты. 
Необходимо подчеркнуть несколько важных моментов в этом подходе.

- В случае $V=a_{4} \phi^{4}$ порядок каждой вершины равен 4. Поэтому двойственный комплекс $D^{*}$ (вершины внутри двумерных клеток $D$ ) имеет двумерные клетки с 4 сторонами. Основной вопрос: дает ли такое перечисление все комплексы с клетками-четырехугольниками (гомеоморфные замкнутым поверхностям) ровно по одному разу?

- Этот вопрос связан с учетом комбинаторных множителей. Деля на $4^{k}$, мы исключаем нумерацию четырех отростков, оставляя их, однако, циклически упорядоченными. Группа автоморфизмов диаграммы факторизуется на два множителя. Первый $C_{v}(D)$ связан с перестановкой вершин, а второй $C_{l}(D)$ относится к перестановкам отростков в отдельных вершинах. Почти все диаграммы имеют тривиальный первый множитель, но для некоторых из них $C_{v}(D)>1$. Можно суммировать по немаркированным диаграммам (как это обычно и делается), но тогда каждая диаграмма приобретет множитель

$$
\frac{C_{l}(D)}{\left.C_{v} D\right)}
$$

Что означает, что данный подсчет не совпадает с естественным, использованным вьше равномерным подсчетом. Строгое доказательство того, что асимптотически разницы не будет, нигде не приводилось.

- Невозможно иметь одновременно положительность коэффициентов как для CMM, так и на множестве получаемых комплексов. Для СММ вероятностное распределение получается только при $a<0$, а на диаграммах положительность достигается при $a>0$. Таким образом, необходимо обосновывать возможность аналитического продолжения от $a<0$ к $a>0$. Поэтому, несмотря на то, что свободная энергия при упомянутом скейлинге может быть явно подсчитана и есть много других строгих результатов, но полное доказательство того, что при упомянутом скейлинге выделяются точно планарные комплексы, отсутствует.

- Есть другие модели чистой гравитации, к которьм применим данный подход: в них явным образом фигурируют числа $n(q, T)$ вершин $v$ с $q_{v}=q$ :

$$
Z=\sum_{T \in \mathbf{A}} \prod_{q>2} t_{q}^{n(q, T)},
$$

где $t_{q}$ - параметры, см. [4].

\section{5. Линейная граничная динамика}

Вероятностная мера

$$
P(T)=Z^{-1} \exp (-\mu F(T))
$$

на некотором классе А комплексов инвариантна относительно следующего простого марковского процесса $T(t)$ со значениями в А. Процесс определяется следующими интенсивностями переходов. С интенсивностью $\lambda_{+}(N), N=F(T(t))$, в момент $t$ мы разбиваем комплекс $T(t)$ на отдельные клетки, добавляем к ним еще одну и склеиваем все клетки заново и случайно, т.е. если $N=F(T(t))$, выбираем $T(t+0)$ согласно 
равномерному распределению на комплексах из класса $\mathbf{A}$ с $N+1$ клетками. С интенсивностью $\lambda_{-}(N)$ мы делаем случайный выбор среди комплексов с $N-1$ клетками.

Если $\lambda_{+}(T)=b f(N), \lambda_{-}(T)=d f(N-1)$ для некоторой положительной функции $f(N)$ и положительных констант $b$ и $d$, то распределение $P(T)$ инвариантно относительно такого процесса. Доказательство состоит из замечания, что индуцированньй на числах $N$ процесс является обратимой цепю Маркова: процессом рождения-гибели на $\mathbb{Z}_{+}$с интенсивностями скачков $q_{i, i+1}=b f(i), q_{i, i-1}=d f(i-1)$, $q_{0,1}=1$. Подобная динамика, однако, не представляет особого интереса.

Простейший способ моделирования Монте-Карло - фиксировать возможно большее $N$ и моделировать равномерное распределение на комплексах с $N$ клетками. Более удобной может быть другая динамика, где $N$ меняется со временем. В оставшейся части статьи мы изучаем локальную динамику. Мы начнем с простейшей локальной динамики, которая хотя и не оставляет нашу меру инвариантной, но тем не менее интересно изучить ее инвариантное распределение и вычислить экспоненту. Мы убедимся, что при этом мы попадаем в другой универсальный класс (т.е. экспонента принимает другое значение).

5.1. Локальный рост. Рассмотрим класс гладких клеточных поверхностей и предположим, что клетки - треугольники. Сначала имеется один треугольник и каждьй шаг состоит в добавлении одной клетки к границе. Могут быть два вида добавления (см. рис. 7): к одному или к двум ребрам с обшей вершиной. К любому ребру на границе прибавляется клетка с интенсивностью $\lambda_{1}$. К любьм двум соседним ребрам на границе прибавляется клетка с интенсивностью $\lambda_{2}$. В любой момент времени комплекс гомеоморфен замкнутому двумерному диску, а его граница - окружности. Предположим, что начальное состояние процесса - одна клетка, предположим также, что если число ребер $m(t)$ границы в момент $t$ равно 3 , то только $\lambda_{1}$-переходы возможны. Можно рассматривать состояния с $m=3$ как триангулящии сферы, где внешность диска отождествляется с грањњю сферы, содержашей северньй полюс. Можно интерпретировать моменты, когда мы в таких состояниях, как закрытие дырки в сфере. В остальные моменты состояние является триангулящией диска.
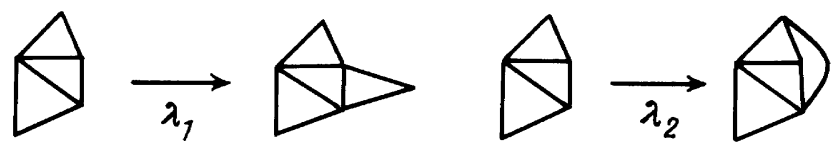

Рис. 7. Динамика на гранище

ЗАмЕчАниЕ 2. Важно заметить, что можно рассматривать два других варианта динамики. Первый - когда мы рассматриваем в качестве множества состояний классы эквивалентности клеточных поверхностей. Тогда интенсивности переходов будут $\lambda_{i} m^{\prime}, i=1,2$, вместо $\lambda_{i} m$, где $m^{\prime}$ равно $m$, деленному на порядок группы автоморфизмов триангулящии диска. Во втором варианте на границе выделяется корневое ребро и переходы возможны, только если они затрагивают это ребро.

Возможны 3 разных типа поведения этого марковского процесса: субкритическое или эргодическое, критическое или нулевое возвратное, суперкритическое или невозвратное. Для всех этих случаев мы изучим поведение локальных коррелящионных 
функций, а также следуюших глобальных переменных в момент $t$ :

$$
F(t)=F(T(t)), \quad V(t)=V(T(t)), \quad L(t)=L(T(t)), \quad m(t)=m(T(t)),
$$

где $L$ - полное число ребер, а $m$ - число ребер на границе.

Субкритический случай. По определению это случай, когда $\lambda_{2}>\lambda_{1}$.

Teоpema 6. Ecлu $\lambda_{2}>\lambda_{1}$, mo

$$
\pi(m=N)=\lim _{t \rightarrow \infty} \mathrm{P}(m(t)=N) \sim_{N \rightarrow \infty} C N^{-1} \exp (-\nu N), \quad \nu=\ln \frac{\lambda_{2}}{\lambda_{1}}
$$

Пусть $\tau$ - случайное число скачков до первого возвращения в состояние $c m=3$, положим $P_{0}(T)=\mathrm{P}(T(\tau)=T)$ для всех триангуляиий диска Т. Тогда

$$
P_{0}(F(T)=N) \sim C\left(\frac{\lambda_{2}-\lambda_{1}}{\lambda_{1}+\lambda_{2}}\right)^{N} .
$$

ДокАЗАТЕльство. Заметим сначала, что длина $m(t)$ границы сама образует марковский процесс: эволюцию гранищы можно рассматривать как грамматику с алфавитом, состоящим из единственного символа 1 (представляющего одно ребро), и с подстановками

$$
\lambda_{1}: 1 \rightarrow 11, \quad \lambda_{2}: 11 \rightarrow 1 \text {. }
$$

Этот процесс очевидным образом сводится к ветвяшемуся процессу с одним типом частищ, где $\lambda_{1}$ - интенсивность рождения, $\lambda_{2}$ - интенсивность гибели. Цепь Маркова с непрерьвным временем $m(t)$, состояниями которой являются точки интервала $[3, \infty)$ решетки, имеет скачки \pm 1 и соответствующие интенсивности $\lambda_{1} k$ из $\lambda_{2} k$ из точки $k \in[3, \infty]$. Ее начальное состояние $m(0)=3$. Стационарная мера процесса $m(t)$ равна

$$
\pi(m=k)=\frac{\prod_{i=3}^{k-1} \lambda_{1} i}{\prod_{i=4}^{k} \lambda_{2} i} \sim C k^{-1} \exp (-\nu k), \quad \nu=-\ln \frac{\lambda_{1}}{\lambda_{2}} .
$$

ЗАмЕчАниЕ 3. Для динамики с локальным наблюдателем (т.е. второго варианта динамики в предыдущем замечании) те же соображения показывают, что

$$
\pi(m=k)=\frac{\prod_{i=3}^{k-1} \lambda_{1}}{\prod_{i=4}^{k} \lambda_{2}} \sim C \exp (-\nu k)
$$

Экспоненты различаются, как и следовало ожидать, на 1.

Рассмотрим процесс скачков (с дискретным временем) $z_{n}=m\left(t_{n}\right)$ для процесса $m(t)$, где $t_{1}<t_{2}<\cdots$ - моменты скачков. Пусть $f(s)=\frac{1}{\lambda_{1}+\lambda_{2}}\left(\lambda_{2}+\lambda_{1} s^{2}\right)-$ производящая функция переходов процесса скачков и $f_{n}(s)$ - ее итерации, пусть $n(\omega)$ - первьй момент, когда $m\left(t_{n}\right)=3$. Известно (см. [30]), что

$$
P(n(\omega)=N)=f_{N}(0)-f_{N-1}(0) \sim C m^{N}, \quad m=\frac{\lambda_{2}-\lambda_{1}}{\lambda_{1}+\lambda_{2}} .
$$


Это и дает доказательство.

Суперкритический случай. Если $\lambda_{1}>\lambda_{2}$, то граница имеет экспоненциальный рост. Набор $(V, L, F, m)$ ведет себя как вырожденньй ветвящийся процесс с 4 типами частиц и со следуюшими интенсивностями:

$$
\begin{aligned}
& \lambda_{1} m: V \rightarrow V+1, L \rightarrow L+2, F \rightarrow F+1, m \rightarrow m+1, \\
& \lambda_{2} m: V \rightarrow V, L \rightarrow L+2, F \rightarrow F+1, m \rightarrow m-1 .
\end{aligned}
$$

Тогда для некоторой случайной величины $\xi$

$$
\begin{aligned}
m(t) & \sim \xi \exp \left(\lambda_{1}-\lambda_{2}\right) t, & F(t) & \sim c_{1} \xi \exp \left(\lambda_{1}-\lambda_{2}\right) t, \\
V(t) & \sim c_{2} \xi \exp \left(\lambda_{1}-\lambda_{2}\right) t, & L(t) & \sim c_{3} \xi \exp \left(\lambda_{1}-\lambda_{2}\right) t,
\end{aligned}
$$

доказательство повторяет аналогичные рассуждения в [7], если принять во внимание формулу Эйлера $V-L+F=1+m$ и $L=\frac{3}{2} F$.

Критический случай и экспоненты. Если $\lambda_{2}=\lambda_{1}$, то процесс $m(t)$ нулевой возвратный. Нельзя говорить о его стационарных вероятностях, но существует бесконечная положительная стационарная мера. Она имеет асимптотику $\pi_{N}=\operatorname{const} N^{\alpha} c^{N} \mathrm{c}$ экспонентой $\alpha=-1$.

Введем другой аналог стационарных вероятностей: рассмотрим $P(T(\tau)=N)$, где $T(\tau)$ - число клеток в момент $\tau$.

Лемма 10. Пусть $\lambda_{1}=\lambda_{2}=\lambda$. Тогда

$$
P(T(\tau)=N) \sim \frac{C}{N^{2}}
$$

Это хорошо известно, см. [30].

ЗАмЕчАнИЕ 4. Экспонента здесь немного отлична от равновесного случая: $\alpha=$ $-2 \neq-\frac{5}{2}$. Более того, здесь возникает следующий эффект. Экспоненты в эргодическом и нулевом возвратном случаях различны: $\alpha=0$ и $\alpha=-2$ соответственно. Иначе говоря, статистическая сумма в критическом случае сходится, но предел статсумм при $\mu \rightarrow \mu_{\text {cr }}$ бесконечен.

Предельные корреляционные функции. Даже в случаях, когда экспоненты отличаются от тех, которые приняты в физике, интересно изучить локальные корреляционные функции, они определяют флуктуации кривизны.

Обозначим через $i$-ю появившуюся вершину. Положим $q_{i}(t)=0$, если $i$ не появилась до момента $t$, и в остальных случаях положим $q_{i}(t)$ равным числу треугольников (или ребер), инцидентных $i$, в момент $t$. Пусть $\tau(i)$ - первый момент, когда появилась вершина $i$.

Tеорема 7. Существуют $\chi_{k}, \sum \chi_{k}=1$, такие, что при $\lambda_{1}>\lambda_{2}$

$$
\lim _{s \rightarrow \infty} \lim _{i \rightarrow \infty} P\left(q_{i}(\tau(i)+s)=k\right) \rightarrow \chi_{k}
$$


Для произвольных вершин $i<j$ обозначим $\rho(i, j)$ начальное расстояние (т.е. расстояние в момент $\tau(j))$ между ними. Существуют такие константы $a, b>0$, что для любых $i<j$

$$
\left|\lim _{s \rightarrow \infty} P\left(q_{i}(\tau(j)+s)=k, q_{j}(\tau(j)+s)=l \mid \rho(i, j)=d\right)-\chi_{k} \chi_{l}\right|<b \exp (-a d) .
$$

Eсли $\lambda_{2} \geqslant \lambda_{1}, m o$

$$
\lim _{s \rightarrow \infty} \lim _{m \rightarrow \infty} P\left(q_{i}(\tau(i)+s)=k \mid m(\tau(i))=m\right) \rightarrow \chi_{k}
$$

Более того, все коррелячионные функции $\chi_{k}$ являются аналитическими функииями $\lambda_{1}, \lambda_{2}$ для всех значений $\lambda_{1}, \lambda_{2}>0$.

ДоКАЗАТЕЛЬСтво. Для вершины $i$ положим $\xi_{i}(s)=q_{i}(\tau(i)+s)$. Заметим, что $\xi_{i}(0)=2$ и число $q_{i}$ увеличивается до тех пор, пока оба смежных этой вершине ребра не будут накрыты одним треугольником. Рассмотрим некоторую вершину $i$ на границе, а также два ребра $(i-2, i-1),(i-1, i)$ слева и два ребра $(i, i+1),(i+1, i+2)$ справа от нее. В пределе бесконечной гранипы имеется пять независимых пуассоновских процессов $\xi_{-2}, \xi_{-1}, \xi_{0}, \xi_{1}, \xi_{2}$. При этом процессы $\xi_{-2}$ и $\xi_{2}$ состоят в одновременном накрытии ребер $(i-2, i-1),(i-1, i)$ или $(i, i+1),(i+1, i+2)$ соответственно. Процессы $\xi_{-1}$ и $\xi_{1}$ состоят в накрытии ребра $(i-1, i)$ или $(i, i+1)$ соответственно. А процесс $\xi_{0}$ состоит в прибавлении треугольника к ребрам $(i-1, i),(i, i+1)$. Таким образом, $\chi_{k}, k \geqslant 2$, равно вероятности того, что пуассоновский процесс с интенсивностью $2 \lambda_{1}+2 \lambda_{2}$ будет иметь в точности $k-2$ скачка до первого скачка независимого от него пуассоновского процесса с интенсивностью $\lambda_{2}$.

Второе утверждение теоремы доказьвается аналогично, если заметить, что процессы $q_{i}(t)$ и $q_{j}(t)$ независимы, пока расстояние между $i$ и $j$ не меньше 4 . Чтобы расстояние стало меньше 4 , должно быть много $\lambda_{2}$-событий "между" $i$ и $j$, что имеет экспоненциально малую вероятность.

Заметим, что для $\lambda_{2} \geqslant \lambda_{1}$ также имеет место (похожее) свойство экспоненциального убывания корреляций. Определим среднюю кривизну

$$
k=\mathrm{E} R=\sum 2 \pi \frac{6-q}{q} \chi_{q}
$$

где сумма берется по всем $q$. Из экспоненциального убывания следует центральная предельная теорема для кривизны.

TEорема 8. При $\lambda_{2}<\lambda_{1}$ случайнье величинь

$$
\frac{\sum_{i} R_{i}-k V(t)}{\sqrt{V(t)}}
$$

сходятся к гауссовскому распределению при $t \rightarrow \infty$. 
5.2. Обратимые граничные процессы. Причины, почему для введенной динамики мы не получили желаемой экспоненты, довольно деликатны. Дадим интуитивное вероятностное объяснение. Рассматриваемая нами инвариантная мера устроена довольно просто: для заданного $N$ все комплексы равновероятны. В то же время простую инвариантную меру можно ожидать, если процесс обратим. Рассмотреннњй выше процесс необратим, и чтобы получить обратимый процесс, надо добавить возможность удаления граней. Например, пусть $\lambda_{1}=\lambda_{2}=\lambda$, и пусть каждьй граничный треугольник может быть удален с интенсивностью $\mu$. Тогда следующее рассуждение показьвает обратимость этого марковского процесса. Пусть $\alpha_{i}$ - комплексы. Рассмотрим замкнутые пути $\alpha_{1}, \alpha_{2}, \ldots, \alpha_{n}, \alpha_{1}$, где каждый $\alpha_{i+1}$ получается из $\alpha_{i}$ добавлением или удалением треугольника на границе. Число добавлений в таком замкнутом пути $\alpha_{1}, \alpha_{2}, \ldots, \alpha_{n}, \alpha_{1}$ должно быть равно числу удалений, т.е. $n / 2$. Обозначим $\lambda_{\alpha \beta}$ интенсивность скачка из $\alpha$ в $\beta$. Тогда

$$
\lambda_{\alpha_{1} \alpha_{2}} \cdots \lambda_{\alpha_{n} \alpha_{1}}=\lambda_{\alpha_{1} \alpha_{n}} \cdots \lambda_{\alpha_{2} \alpha_{1}}=(\lambda \mu)^{n / 2}
$$

Однако удаление может вывести нас за рассматриваемый класс комплексов. Например, удаление нескольких треугольников может сделать комплекс несвязным. Поэтому надо предположить, что удаление невозможно, если результируюший комплекс не принадлежит нашему классу А комплексов. Такое “граничное” множество состояний имеет довольно сложную структуру, и трудно использовать стандартную процедуру получения стационарных вероятностей с помошью уравнений баланса. Однако если допустить несвязные комплексы, но оставить процесс неприводимым, то это ведет к (нелокальной) операции объединения связных компонент. Именно эта последняя операция приводит нас к нелинейным марковским процессам, которые мы сейчас рассмотрим.

\section{6. Нелинейная граничная динамика}

В предыдущем разделе рассматривалась марковская динамика, состояниями которой были комплексы. Эта динамика была локальной и однородной на гранище. Это не дало нам необходимой экспоненты. Здесь мы построим динамику, дающую в точности экспоненту $\alpha=-\frac{7}{2}$. Однако динамика не будет процессом Маркова. Можно представлять ее как систему независимых частищ (или Вселенных), взаимодействуюших друг с другом, но это не будет марковской бесконечной системой частиц. Простейшее парное взаимодействие (склеивание) двух комплексов даст квадратичное функциональное уравнение и в результате нужную экспоненту.

6.1. Квадратичные квазипроцессы. Пусть $\Delta$-множество вероятностных мер на некотором пространстве $S$. Рассмотрим класс преобразований $M: \Delta \rightarrow \Delta$, обобщающих цепи Маркова. Эти преобразования не порождаются случайными отображениями $S \rightarrow S$, они нелинейны на $\Delta$.

Пусть $S$ не более чем счетно, и пусть цепь Маркова определена на пространстве состояний $S$ с переходными вероятностями $p_{\alpha \beta}, \sum_{\beta: \beta \neq \alpha} p_{\alpha \beta}=1$, из $\alpha$ в $\beta$. Это определяет линейное отображение $L$

$$
q=\left\{q_{\beta}\right\} \rightarrow L q=\left\{\sum_{\alpha} q_{\alpha} p_{\alpha \beta}\right\}
$$


на $\Delta$. Пусть также задано вероятностное ядро

$$
P((\alpha, \gamma) \rightarrow \beta): S \times S \rightarrow S, \quad \sum_{\beta} P((\alpha, \gamma) \rightarrow \beta)=1
$$

Предполагается, что оно симметрично

$$
P((\alpha, \gamma) \rightarrow \beta)=P((\gamma, \alpha) \rightarrow \beta) \quad \text { и } P((\alpha, \gamma) \rightarrow \gamma)=0 .
$$

Оно может быть даже детерминистическим. Оно определяет квадратичное преобразование $Q$ на $\Delta$

$$
q=\left\{q_{\alpha}\right\} \rightarrow Q q=\left\{\sum_{\beta, \gamma} q_{\beta} q_{\gamma} P((\beta, \gamma) \rightarrow \alpha)\right\} .
$$

Рассматривая выпуклую комбинацию, мы имеем преобразования (формально $\left.c_{0}+c_{1} L+c_{2} Q\right)$ на классе мер на $S$

$$
\begin{aligned}
q(\alpha, t+1)= & r_{1} \sum_{\beta} q(\beta, t) p_{\beta \alpha} \\
& +r_{2} \sum_{\beta, \gamma} q(\beta, t) q(\gamma, t) P((\beta, \gamma) \rightarrow \alpha)+\left(1-r_{1}-r_{2}\right) c_{0}(\alpha),
\end{aligned}
$$

где $c_{0}(\alpha)$ - некоторая вероятностная мера, а неотрицательные числа $r_{1}, r_{2}$ таковы, что $0 \leqslant r_{1}+r_{2} \leqslant 1$. При этом полная масса $\sum_{\alpha} q(\alpha)=1$ сохраняется.

Это можно интерпретировать так. Рассмотрим счетное число частиц на $S, q_{\alpha}-$ среднее число частиц в точке $\alpha$. С вероятностью $r_{1}$ каждая частица (независимо от других) делает скачок в соответствии с вероятностями $p_{\alpha \beta}$. Это дает линейное преобразование. $\mathrm{C}$ вероятностью $r_{2}$ частицы образуют пары так, что среднее число пар $(\alpha, \beta)$ является произведением средних, тогда каждая пара $(\alpha, \gamma)$, независимо от других, дает рождение одной частище в точке $\beta$ с вероятностью $P(\alpha, \gamma \rightarrow \beta)$. Кроме того, с вероятностью $1-r_{1}-r_{2}$ происходит иммиграция со средним $c_{0}(\alpha)$ в точки $\alpha$. Следует подчеркнуть, что нет процесса в обычном смысле, но только преобразование мер. Это кажется связанным с теорией поля для струн (вторичным квантованием струн), но последняя физическая теория не имеет пока математического статуса.

Немного общей теории. Общей теории таких квадратичных процессов не существует, но какой-то минимум ее нужен. Удобно записьвать их в виде

$$
W=p_{1} W_{1}+p_{2} W_{2}+\left(1-p_{1}-p_{2}\right) W_{0} .
$$

Пусть $k_{1}$ - коэффищиент сжатия для $W_{1}$, т.е. такое число, что для произвольных вероятностных мер $\mu_{1}, \mu_{2}$ имеем $\left\|W_{1}\left(\mu_{1}-\mu_{2}\right)\right\| \leqslant k_{1}\left\|\mu_{1}-\mu_{2}\right\|$. Пусть $k_{2}(x)$ - коэффициент сжатия для стохастической матрищы $P_{y z}(x)=P((x, y) \rightarrow z)$ и $k_{2}=\sup _{x} k_{2}(x)$.

ТЕОРема 9. Пусть $p_{1} k_{1}+2 p_{2} k_{2}<1$. Если число состояний конечно, то существует точно одна неподвижная точка отображения $W$, причем сходимость к ней экспоненииальная. Если число состояний счетно, то это утверждение имеет место при условии, что существует функиия (Ляпунова) $f(x)$ такая, что $\sum_{x} f(x)<\infty$ иесли $\mu(x) \leqslant f(x)$, то $(W \mu)(x) \leqslant f(x)$. 
ДокАЗАТЕЛЬСтво. Рассмотрим две вероятностные меры $\nu$ и $\mu=\nu+\varepsilon$. Тогда мы получим следуюшее свойство сжатия для $\rho(\nu, \mu)=\|\varepsilon\|$ :

$$
\begin{aligned}
\rho(W \mu, W \nu) & =\left\|p_{1} W_{1} \varepsilon+p_{2} \sum \mu(x) \varepsilon(y) P((x, y) \rightarrow z)+p_{2} \sum \nu(x) \varepsilon(y) P((x, y) \rightarrow z)\right\| \\
& \leqslant\left(p_{1} k_{1}+2 p_{2} k_{2}\right)\|\varepsilon\| .
\end{aligned}
$$

Отсюда следует первое утверждение теоремы. Для доказательства второго заметим, что ввиду компактности существует неподвижная точка $\nu$ в $A=\{\mu: \mu(x) \leqslant f(x)\}$ и для каждой $\mu_{0}$ последовательность $W^{n} \mu_{0}$ сходится к $\nu$.

Квазипроцессы с непрерывньм временем определяются аналогично. Вместо вероятностей $p$ мы вводим интенсивности $\lambda$ : с интенсивностью $\lambda_{1}$ мы делаем линейное преобразование, с интенсивностью $\lambda_{2}$ квадратичное, и иммиграция происходит с интенсивностью $\lambda_{0}$. Уравнения для стационарной меры следующие:

$\left(\lambda_{1}+\lambda_{2}+\lambda_{0}\right) \pi(\alpha)=\lambda_{1} \sum_{\beta} \pi(\beta, t) p_{\beta \alpha}+\lambda_{2} \sum_{\beta, \gamma} \pi(\beta, t) \pi(\gamma, t) P((\beta, \gamma) \rightarrow \alpha)+\lambda_{0} c_{0}(\alpha)$.

Временная эволюция управляется уравнением

$$
\begin{aligned}
\frac{d q(\alpha, t)}{d t}= & \lambda_{1} \sum_{\beta}(q(\beta, t)-q(\alpha, t)) p_{\beta \alpha} \\
& +\lambda_{2} \sum_{\beta, \gamma}(q(\beta, t) q(\gamma, t)-q(\alpha, t)) P((\beta, \gamma) \rightarrow \alpha)+\lambda_{0}\left(c_{0}(\alpha)-q(\alpha, t)\right) .
\end{aligned}
$$

6.2. Производящие функции. Возьмем теперь в качестве $S$ множество всех корневых триангуляций диска (с корнем на границе). Возьмем некоторую вероятностную меру $\mu_{0}$ на $S$ в качестве начальной меры и пусть динамика задается движениями Тутте, изображенными на рис. 5 . В качестве меры $c_{0}(\alpha)$ возьмем единичную меру на комплексе с $N=0, m=2$, состоящем из одного ребра и двух вершин. Удобно будет считать его принадлежашим нашему классу. Вся случайность будет заключена в вероятностях $p_{0}, p_{1}, p_{2}$, а “линейные" скачки и стохастическое ядро будут детерминированными:

$$
\begin{gathered}
p_{(N, m),(N+1, m-1)}=1, \\
P\left(\left(\left(N_{1}, m_{1}\right),\left(N_{2}, m_{2}\right)\right) \rightarrow\left(N_{1}+N_{2}+1, m_{1}+m_{2}-1\right)\right)=1, \quad c_{0}((0,2))=1 .
\end{gathered}
$$

Если начальная мера $\mu_{0}$ такова, что значение меры $\mu_{0}(T)$ на любом комплексе $K$ зависит лишш от $N=N(K), m=m(K)$, то все итерации $T^{n} \mu_{0}$ меры $\mu_{0}$ обладают этим свойством. Мы будем это всегда предполагать и докажем ниже, что предел итераций $T^{n} \mu_{0}$ сушествует и является единственным. В действительности можно показать, что то же имеет место для любой начальной вероятностной меры, так как она "выметается" динамикой из любого конечного подмножества $S$. 
При данном предположении можно рассматривать проекцию этой динамики на $\mathbb{Z}_{+}^{2}$, где точки $\mathbb{Z}_{+}^{2}$ обозначаются $\alpha=(N, m), N$ - число граней и $m$ - число ребер гранищы. Таким образом, мера точки $(N, m)$ равна сумме мер соответствующих комплексов. Оказьвается, что эта проекция - такой же квадратичный квазипроцесс. Введем производящую функцию

$$
U(x, y)=\sum_{N, m=0}^{\infty} q(N, m) x^{N} y^{m} .
$$

Заметим, что имеет место следуюшее условие однородности: для всех $\alpha, \beta, \alpha_{1}, \beta_{1}, \gamma$ таких, что все $\alpha, \beta, \alpha+\gamma, \beta+\gamma, \alpha_{1}, \beta_{1}, \alpha_{1}+\beta_{1}+\gamma$ принадлежат четверти-плоскости,

$$
p_{\alpha, \alpha+\gamma}=a_{\gamma}, \quad P((\alpha, \beta) \rightarrow \alpha+\beta+\gamma)=P\left(\left(\alpha_{1}, \beta_{1}\right) \rightarrow \alpha_{1}+\beta_{1}+\gamma\right)=b_{\gamma} .
$$

Инвариантная мера $q(N, m)$ динамики определяется, таким образом, системой рекуррентных уравнений

$$
\begin{aligned}
q(N, m)= & r_{1} q(N-1, m+1)+r_{2} \sum_{N_{1}+N_{2}=N-1, m_{1}+m_{2}=m+1} q\left(N_{1}, m_{1}\right) q\left(N_{2}, m_{2}\right) \\
& +r_{0} \delta_{(N, m),(0,2)}
\end{aligned}
$$

и граничньгми условиями

$$
C(N, 0)=C(N, 1)=0, \quad C(0,2)=1, \quad C(0, m)=0, \quad m \neq 2 .
$$

Умножая на $x^{N} y^{m}$ и складьвая, получаем уравнение для производяших функций

$$
U=r_{1} U x y^{-1}+r_{2} U^{2} x y^{-1}+\left(1-r_{1}-r_{2}\right) y^{2}-x y^{-1} r_{1} \sum_{N} q(N, 2) x^{N} y^{2} .
$$

В нем удобно сделать скейлинг

$$
x \rightarrow x r_{1}^{-1}, \quad q(N, m) \rightarrow a q(N, m) .
$$

Если положить $a r_{2}=1, \beta=\frac{\left(1-r_{1}-r_{2}\right) r_{2}}{r_{1}}$, то получим

$$
U=U x y^{-1}+U^{2} x y^{-1}+\beta y^{2}-x y^{-1} \sum_{N} q(N, 2) x^{N} y^{2} .
$$

Назовем это уравнение каноническим. Заметим, что случай $\beta=1$ или $r_{1}=\frac{r_{2}\left(1-r_{2}\right)}{1+r_{2}}$ соответствует проблеме перечисления комплексов и был полностью разобран Тутте. Здесь мы фактически воспроизводим его анализ в более общей ситуации.

Если существует решение $U$ канонического уравнения с неотрицательными коэффишиентами $q(N, m)$, то инвариантная мера $\widetilde{q}(N, m)$ для динамики равна $\widetilde{q}(N, m)=$ $a^{-1} r_{1}^{N} q(N, m)$.

Мы ограничиваемся классом $E$ инвариантных мер, удовлетворяюших следуюшим условиям:

1) (экспоненциальные оценки) $q(N, m)<C^{N+m}$ для некоторого $C>0$;

2) $q(N, m)$ неотрицательны.

Главный результат составляет следуюшая теорема. 
ТЕОрема 10. Для всех $r_{1}, r_{2}$ существует единственная положстельная инвариантная мера. Она конечна, если и только если $\frac{2\left(1-r_{1}-r_{2}\right) r_{1} r_{2}}{27}<1$.

Теорема вытекает из следующей леммы, если учесть сделанньй скейлинг.

Лемма 11. Для всех $\beta$ существует единственное решение канонического уравнения с положительными коэффициентами в рассматриваемом классе мер. Ряд $\sum_{N, m} q(N, m)$ сходится тогда и только тогда, когда $\sqrt{2 /(27 \beta)}>1$.

6.2.1. Анализ функционального уравнения. Здесь мы докажем основную лемму. Полагая $U=y^{2} W$ и определяя $S_{m}(x)$ посредством

$$
W(x, y)=\sum_{m=2}^{\infty} S_{m-2}(x) y^{m-2},
$$

можно переписать функциональное уравнение в виде

$$
W=\beta+x y W^{2}+x y^{-1}(W-S), \quad S(x)=S_{0}(x)=W(x, 0) .
$$

Если $S_{0}(x)$ известен как формальный ряд, то все $S_{m}$ находятся из него последовательно

$$
y S_{0}=\beta y+y x S_{1}, \quad y^{2} S_{1}=y^{2} x S_{0}^{2}+y^{2} x S_{2}, \ldots .
$$

Мы частично следуем выводу из [22]. Перепишем функциональное уравнение (7) в виде

$$
(2 x U+x-y)^{2}=4 x^{2} y^{2} S+(x-y)^{2}-4 \beta x y^{3}
$$

и обозначим его правую часть через $D$. Рассмотрим аналитическое множество $\{(x, y)$ : $2 x U+x-y=0\}$ в малой окрестности $x=y=0$. Заметим, что оно непусто, содержит $(0,0)$, оно определяет функцию $y(x)=x+O\left(x^{2}\right)$ в окрестности $x=0$. Докажем, что $y(x)$ и $S(x)$ - алгебраические функции. Мы имеем два уравнения, справедливых в точках этого аналитического множества:

$$
D=0, \quad \frac{\partial D}{\partial y}=0
$$

или

$$
\begin{aligned}
& 4 x^{2} y^{2} S(x)+(x-y)^{2}-4 \beta x y^{3}=0 \\
& 8 x^{2} y S(x)-2(x-y)-12 \beta x y^{2}=0
\end{aligned}
$$

откуда можно получить $y(x)$ и $S(x)$ :

$$
x=y\left(1-2 \beta y^{2}\right), \quad S=\frac{\beta\left(1-3 \beta y^{2}\right)}{\left(1-2 \beta y^{2}\right)^{2}} .
$$

Алгебраическая функция $y(x)$ удовлетворяет уравнению $y^{3}+p y+q=0$ c

$$
p=-\frac{1}{2 \beta}, \quad q=\frac{x}{2 \beta} .
$$


Дискриминант

$$
\Delta=-4 p^{3}-27 q^{2}=p^{2}\left(\frac{2}{\beta}-27 x^{2}\right)
$$

не является к вадратом в поле рациональных функций. Тогда (см. [31]) группа Галуа есть $S_{3}$ и точки ветвления равны $x= \pm \sqrt{2 /(27 \beta)}$. Выпишем (формальное) решение Кардано кубического уравнения

$$
y=\sqrt[3]{-\frac{q}{2}+\sqrt{\frac{q^{2}}{4}+\frac{p^{3}}{27}}}+\sqrt[3]{-\frac{q}{2}-\sqrt{\frac{q^{2}}{4}+\frac{p^{3}}{27}}} .
$$

Заметим, что

$$
\left|\frac{q}{2}\right| \neq\left|\sqrt{\frac{q^{2}}{4}+\frac{p^{3}}{27}}\right|
$$

и, таким образом, внутри круга радиуса $x_{1}=\sqrt{2 /(27 \beta)}$ нет сингулярностей. Нам нужна ветвь с $y(0)=0$. Так как $y\left(x_{1}\right)>0$, то в случае нулевого дискриминанта имеет место $y\left(x_{1}\right)=\sqrt[3]{x_{1} /(2 \beta)}$. Итерируя уравнение

$$
y=\frac{x}{1-2 \beta y^{2}},
$$

мы получим, что разложение $y(x)$ в точке $x=0$ имеет положительные коэффициенты. Ведушая сингулярность $y(x)$ есть $\left(x-x_{1}\right)^{1 / 2} . S$ является алгебраической функцией, аналитической при $|x|<\sqrt{2 /(27 \beta)}$. Действительно, $S$ могла бы иметь полюс в $|x|<$ $\sqrt{2 /(27 \beta)}$, только если $1-2 \beta y^{2}(x)=0$, но отсюда следовало бы, что $x=0$, что невозможно. Обозначим $y_{0}(x)=y(x)$ и подставим

$$
S=\frac{\beta\left(1-3 \beta y_{0}^{2}(x)\right)}{\left(1-2 \beta y_{0}^{2}(x)\right)^{2}}, \quad x=y_{0}(x)\left(1-2 \beta y_{0}^{2}(x)\right)
$$

в (8). В разложении $S$ два члена с противоположными знаками дают сокращение сингулярности наименьшего порядка $\left(x-x_{1}\right)^{1 / 2}$ у функции $y_{0}(x)$, и поэтому мы имеем для $S$ ведушую сингулярность $\left(x-x_{1}\right)^{3 / 2}$. Чтобы представить разложение $U$ явно, напишем

$$
\begin{aligned}
D & =4 y^{2} y_{0}^{2}\left(1-3 \beta y_{0}^{2}\right)+\left(y_{0}(x)\left(1-2 \beta y_{0}^{2}(x)\right)-y\right)^{2}-4 \beta y^{3} y_{0}(x)\left(1-2 \beta y_{0}^{2}(x)\right) \\
& =\left(y-y_{0}\right)^{2}(a(x)+b(x) y),
\end{aligned}
$$

так как $y=y_{0}(x)-$ двойной корень основного уравнения, и

$$
a(x)=\left(1-2 \beta y_{0}^{2}(x)\right)^{2}, \quad b(x)=-4 \beta y_{0}(x)\left(1-2 \beta y_{0}^{2}(x)\right) .
$$

Выбирая знак минус, получим

$$
U(x, y)=\frac{y-x}{2 x}-\frac{\sqrt{D}}{2 x}=\frac{-x+y_{0}(x)}{2 x}-\left(y-y_{0}(x)\right) \frac{\sqrt{a(x)+b(x) y}-1}{2 x},
$$

что дает законное разложение.

Для данного $x$ радиус сходимости $U$ как функции $y$ определяется нулями $\sqrt{a(x)+b(x) y}$ или $\sqrt{1-\frac{4 \beta y_{0}^{2}}{x} y}$. Так как $\frac{y_{0}^{2}}{x}$ возрастает на интервале $\left[0, x_{1}\right]$, то радиус сходимости $U$ для $x=x_{1}$ равен $R=\frac{x_{1}}{4 \beta y_{0}^{2}\left(x_{1}\right)}$. 
6.3. Корреляционные функции. Используя вводимое нижепредставление комплексов деревьями, мы докажем, что для большинства вершин условные распределения случайных величин $q_{v}$ сходятся к единственному пределу, если $N \rightarrow \infty$. Мы используем для этого специальный прием выделения вершин $v$ в рекуррентно строяшемся комплексе. Мы обсуждали эту проблему в [6], [7] в более общей ситуации. Доказательство, имеюшее комбинаторный неаналитический характер, довольно сложное и мы излагаем его неформально.

6.3.1. Представление комплексов деревьями. Процесс построения комплекса последовательным применением подстановок Тутте будет представлен в виде плоского дерева. Это даст взаимно однозначное соответствие между комплексами и некоторым классом планарных деревьев. Плоское дерево растет из своей корневой вершины вверх (это показано на рис. 8 направлением стрелок). Пусть $\mathbf{A}_{0}-$ класс корневых плоских триангуляший. Мы будем обозначать вершины деревьев через $v$, а вершины комплексов через $w$.
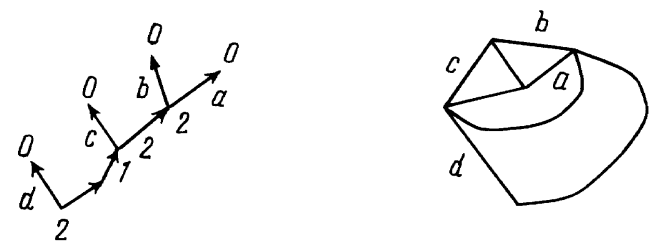

Рис. 8. Плоские деревья и карты

Введем класс $\mathbf{T}_{0}$ плоских деревьев, определяемый следуюшим образом. Каждое дерево из этого класса может иметь три типа вершин: $0,1,2$ согласно числу ребер, идуших вверх от вершины. Вершины типа 0 также назьваются концевыми вершинами. Пусть $n_{i}$ - число вершин типа $i$. Среди 2-вершин (т.е. вершин типа 2) есть вершины, иншидентные (т.е. связаны одним ребром) ровно одной 0 -вершпне, обозначим их число $n_{20}$, а число тех, которые имеют две инцидентных 0-вершины, обозначим $n_{00}$. Для любой вершины $v$ обозначим $T_{v}$ дерево, состояшее из вершины $v$ и всех вершин над $v$. Обозначим $n_{i}(v)$ и т. д. соответствуюшие числа для деревьев $T_{v}$. Заметим, что $n_{0}(v)=n_{2}(v)+1$. Тогда класс $\mathbf{T}_{0}$ характеризуется следующим ограничением: $n_{0}(v)-n_{1}(v)-1 \geqslant 0$ для всех вершин $v$ типа 1.

ЛЕмма 12. Описываемая ниже конструкиия дает взаимно однозначное соответствие межсду $\mathbf{A}_{0} u \mathbf{T}_{0}$.

Покажем, что каждьй комплекс порождает плоское дерево из $\mathbf{T}_{0}$, иначе говоря, существует функция $f$ из множества комплексов в множество плоских деревьев. Докажем это индукцией по числу граней. Сам комплекс представим корневой вершиной дерева. Мы уменьшаем комплекс обратными подстановками Тутте, при этом мы тянем вверх одно ребро в случае подстановки типа 1 и 2 ребра в случае подстановки типа 2, разбивающей комплекс на два. Эти два комплекса мы сопоставляем правой или левой вершинам согласно ориентации корневого ребра комплекса следующим образом. Корневые ребра обоих комплексов имеют одну ориентацию и одно следует за другим. Комплекс с предшествуюшим корневым ребром мы сопоставляем левой вершине, а с последуюшим - правой. На каждом шаге число граней уменњшается на 1 , 
и далее мы поступаем по индукции. Когда мы получаем комплекс из одного ребра (комплекс-ребро), соответствуюшая вершина будет концевой вершиной дерева.

Заметим, что $N=n_{1}+n_{2}, L=n_{0}+n_{1}+n_{2}, V=n_{0}+1, m=n_{0}-n_{1}+1$. Здесь $N, L, m, V$ относятся к комплексу, а числа $n_{i}, n_{00}$ и т. д. - к дереву. Для доказательства последнего равенства заметим, что каждая 1-вершина и каждая 2-вершина (кроме инцидентных хотя бы одной 0-вершине) уменшают $m$ на единицу, каждая из $n_{20}$ вершин увеличивает $m$ на единицу, и каждая из $n_{00}$ вершин увеличивает $m$ на три. Тогда $m=n_{20}+3 n_{00}-n_{1}-\left(n_{2}-n_{00}-n_{20}\right)=n_{0}-n_{1}+1$. Так как $m \geqslant 2$ для рассматриваемых комплексов, мы получаем введенное ограничение.

Покажем теперь, что каждое плоское дерево класса $\mathbf{T}_{0}$ порождает комплекс - т.е. покажем, что $f$ взаимно однозначна. Возьмем дерево и будем переходить по индукции от верхних вершин к нижним. Все концевые вершины объявим комплексами-ребрами. Возьмем $V$ комплексов-ребер, занумеруем их слева направо $v=1,2, \ldots, V$ как концевые вершины дерева. Эти ребра дадут $V+1$ вершин в комплексе. Отметим эти концевые вершины. На каждом шаге индукции возьмем неотмеченную вершину $v$ такую, что над ней есть только отмеченные вершины. Если $v$ имеет тип $i$, то на данном шаге индукции применяем подстановку Тутте типа $i$. После этого шага мы отмечаем $v$. Обратно, комплекс, полученный подобньм образом, отображается функцией $f$ на дерево, с которого мы начали. Все комплексы, возникшие в процессе индукции, законны, так как $m \geqslant 2$. Лемма доказана.

Вклад дерева $G(T)$ определяется как произведение $r_{0}^{n_{0}} r_{1}^{n_{1}} r_{2}^{n_{2}}$, где $n_{i}=n_{i}(T)$ число вершин типа $i$, что равно конечно вероятности соответствуюшего корневого комплекса.

ЗАМЕЧАНИЕ 5. Плоские деревья находятся в соответствии с системами скобок, которые могут быть расставлены в произведении $a_{1} \cdots a_{n}$ в неассоциативной некоммутативной алгебре, см. рис. 9. Однако введенное ограничение на $\mathbf{T}_{0}$ делает ситуацию более сложной.

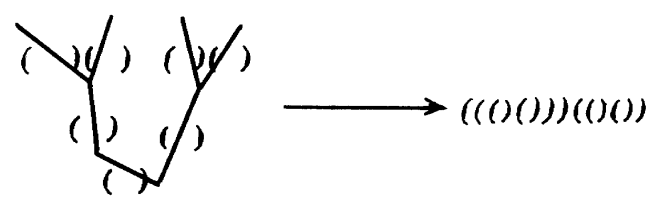

Рис. 9. Планарные деревья и скобки

6.3.2. Локальная кривизна. Возьмем конщевую вершину $v$ и положим $l(v)=$ $\max (v, V-v)$.

Teорема 11. Пусть $l(v) \rightarrow \infty($ тогда необходимо $V \rightarrow \infty u N \rightarrow \infty)$. Тогда для равновесного распределения $P$ существует предел

$$
\lim P\left(q_{v}=k\right)=p(k), \quad \sum_{k=2}^{\infty} p(k)=1 .
$$


ДокАЗАТЕЛЬство. Каждой концевой вершине соответствует некоторый комплекс, состоящий из одного ребра. Их число равно $V$. Будем представлять эти ребра положенными горизонтально на горизонтальную прямую и ориентированными слева направо. Возьмем некоторую концевую вершину $v$ и левую (т.е. корневую) вершину $w=w(v)$ соответствуюшего комплекса-ребра. Ориентацию комплекса будем считать против часовой стрелки. Определим историю каждой вершины $w(v)$. Эта история может описьваться как в терминах комплекса, так и в терминах дерева, и будет удобно использовать оба описания. По определению она состоит из нескольких частей, этапов истории, единственного пути по дереву из $v$ к корню дерева $T$ в направлении вниз. С точки зрения комплексов история заканчивается или когда вершина $w(v)$ исчезает с гранишы комплекса (это означает, что $q_{w}$ не будет больше меняться), или в корне дерева, если $w$ остается на гранище комплекса. Первая часть истории длится, пока вершина не будет покрыта ребром. $q_{w}$ может меняться только на таких кусках истории. С точки зрения деревьев первая часть истории начинается с концевой вершины $v$, спускается вниз от вершины к вершине и продолжается до первой встреченной правой 2-вершины. Мы говорим, что 2-вершина есть правая вершина, если мы (т.е. вершина $w)$ пришли в нее справа, т.е. $w(v)$ лежит в правом комплексе. Следующая часть истории начинается, когда $w(v)$ становится принадлежащей корневому ребру комплекса, и кончается так же, как и первая часть.

Если вершина $a$ дерева находится под $v=w(v)$, то $w$ принадлежит соответствуюшему комплексу $f^{-1}(a)$. Обозначим $a(w)$ наименьшее $a$ такое, что $a<v$ и $w$ на границе $f^{-1}(a)$.

Обозначим $b(w)$ наименьшее $b$ такое, что $b<v$ и $w$ является корневой вершиной соответствуюшего комплекса для каждого $b_{i}$ из интервала $v>b_{1}>b_{2}>\cdots>b$ на дереве. Длина $L(v(w))$ этого интервала (пути) на дереве в точности равна разности $q_{w}(j)-q_{w}(k)$, где $j$ - первьй комплекс пути (т.е. комплекс-ребро), $k$ - последний. Мы докажем, что $P(L(v(w))=n)<C \exp (-\gamma n)$. Заметим, что для доказательства достаточно рассматривать произвольную инвариантную меру, так как для любой из них условные вероятности при фиксированных $N, m$ одинаковы (в основном нам интересен лишь случай $m=3$ ).

Если удалить из дерева все 1-вершины, то результируюшее дерево без 1-вершин будем называть голым деревом. Оно определяет класс эквивалентности одетых деревьев, каждое из которых может быть получено добавлением некоторого числа 1-вершин к голому дереву. Каждая неотрицательная мера на множестве деревьев индуцирует меру на классах эквивалентности - т.е. на голых деревьях. Начнем с голых деревьев.

Случай голых деревьев. Если 1-вершин нет вообще, то $m=n_{0}+1$, число граней также равно $m$. В этом случае все вершины принадлежат границе комплекса, и после того как вершина покрывается ребром, она больше не участвует в процессе, т.е. ее история состоит из одной части. При этом она покрьвается ребром, только когда к комплексу, в котором находится $w$, что-то подсоединяется слева, а до тех пор могут быть, скажем, $k$ подсоединений справа, которые дадут $k$ дополнительных ребер в $q_{w}$. При этом правое соединение присоединяет к $w$ комплекс, соответствующий некоторому дереву, покрьвающему интервал справа от $v$.

Оценим для примера вероятность $P\left(k ; j_{1}, \ldots, j_{k}\right)$ того, что есть ровно $k$ подсоединений интервалов длины $j_{1}, \ldots, j_{k}$, покрываюших интервал $I_{r}=\{v+1, \ldots, v+R\}$, до левого подсоединения, покрьвающего интервал $I_{l}=\{v-L, \ldots, v-1\}$ для некоторого 
$L>0$. Мы используем следуюшее марковское свойство. Назовем вершину $v^{\prime}=v(I)$ $I$-разделяюшей, если она покрьвает в точности интервал $I$. Тогда число деревьев с разделяющей вершиной $v^{\prime}$ равно $t(I) t\left(V-I+v^{\prime}\right)$, где $t(I)$ - число деревьев на интервале $I$, так как $t\left(V-I+v^{\prime}\right)$ есть также число фактор-деревьев по отношению к множеству поддеревьев на $I$. Таким образом, вероятность, что $v^{\prime}$ является $I$-разделяюшей, может быть оценена как

$$
\frac{t(I) t\left(V-I+v^{\prime}\right)}{t(V)}<\min \left(b, c I^{-3 / 2}\right),
$$

где $t(n+1)=\frac{1}{n+1} C_{2 n}^{n} \sim c n^{-\frac{3}{2}} 2^{n}-$ известные числа Каталана и $b<1$. Отсюда, в частности, следует, что вероятность $P_{v}$ того, что правое соединение с вершиной $v$ возникнет раньше, чем левое, не превосходит некоторой константы $d<1$.

Поэтому с помощью марковского свойства по индукции получим для достаточно больших $r$ и некоторого $a<1$

$$
P\left(q_{v} \geqslant r\right)<a^{r} .
$$

Мы получили экспоненциальные оценки. Существование термодинамического предела следует из того, что влияние границ сказывается только с вероятностями, меншшими $a^{l(v)}$.

Случай $n_{1}>0$. Здесь мы фиксируем голое дерево, рассматриваем одну задачу о распределении 1-вершин на голом дереве и получаем оценки, равномерные по голым деревьям.

Оценим сначала вероятность того, что вершина $w$ приобретет большое $q_{w}$ за счет 1-вершин до того, как она будет впервые покрыта. Мы будем делать оценки на каждой части истории отдельно и рассмотрим подробно только первую часть истории. Пусть $v$-вершина дерева, где она кончается. Пусть $T_{v}$ - дерево над этой вершиной с корнем в $v$ и $f^{-1}(v)$ - соответствуюший комплекс.

Начнем со случая, когда в $T_{v}$ есть только одна 00-вершина, тогда 0-вершины последовательно присоединяются к уже сушествуюшим деревьям. Значит, возможные способы расположения 1-вершин можно характеризовать как число упорядоченных наборов неотрицательных целых чисел $a_{1}, \ldots, a_{n}$ таких, что для всех $k=1,2, \ldots, n$ будет $\sum_{i=1}^{k} a_{i} \leqslant k-2$.

Удобно сформулировать следуюшую абстрактную урновую задачу. Пусть имеется $n$ урн и $m$ шаров, которые размешены в урнах. Пусть $a_{i}$ - число шаров в урне $i$. Пусть $c(n, m)$ - число расположений $a_{1}, \ldots, a_{n}$ шаров с условиями $\sum_{i=1}^{k} a_{i} \leqslant k$, $\sum_{i=1}^{m} a_{i}=m \leqslant n$. Имеет место рекуррентное соотношение

$$
c(n, m)=\sum_{k=0}^{m} c(n-1, m-k)
$$

или

$$
c(n, m)=c(n, m-1)+c(n-1, m-1),
$$

из которого нетрудно извлечь асимптотику для числа $c(n, m ; i)=c(n-1, m-i)$ расположений среди $c(n, m)$ таких, что в последней урне ровно $i$ шаров. Нетрудно получить формулу для производящей функции

$$
f(x, y)=\sum_{n=-\infty}^{\infty} \sum_{m=1}^{\infty} c(n, m) x^{n} y^{m}=\frac{y}{1-y(1+x)} \sum_{k=1}^{\infty} k x^{k},
$$


коэффициенты которой совпадают с нашими для $n \geqslant m$. Мы хотим доказать, что $\frac{c(n, m)}{c(n, m-1)}<b$ для некоторого $b<1$, и найти метод (отличньй от метода производящих функций), которьй действовал бы в более общей ситуации. Выразим сначала $c(n, m)$ через число путей от прямой $m=1 \mathrm{k}$ точке $(n, m)$. При этом шаг пути может быть либо $(0,1)$, либо $(1,1)$. Имеем

$$
c(n, m)=\sum_{k=m+1}^{n} k L(k ; n, m)
$$

где $L(k ; n, m)$ - число путей из точки $(k, 1)$ в точку $(n, m)$, так как $c(k, 1)=k$. Так как $L(k ; n, m-1)=L(k-1 ; n-1, m-1)$, то $\frac{c(n, m-1)}{c(n-1, m-1)}=1+O(1 / m)$. Отсюда и следует результат.

Переходим теперь к обшему случаю. Вместо урновой задачи на отрезке $[1, n]$ мы имеем урновую задачу на произвольном плоском дереве $T$ с условием

$$
\sum_{i \in T_{v}} a_{v} \leqslant V\left(T_{v}\right), \quad \sum_{v \in T} a_{v}=m
$$

где $a_{v}$ - число шаров в урне (вершине) $v$ дерева, $V\left(T_{v}\right)$ - число вершин у дерева $T_{v}$. Пусть $c\left(T_{v}, m\right)$ - число расположений на дереве $T_{v}$ с $m$ шарами. Если, например, от вершины $v$ идут вверх два ребра в вершины $v(1), v(2)$, то

$$
c\left(T_{v}, m\right)=\sum_{i=0}^{m} c\left(T_{v}, m ; i\right), \quad c\left(T_{v}, m ; i\right)=\sum_{m_{1}+m_{2}=m-i} c\left(T_{v(1)}, m_{1}\right) c\left(T_{v(2)}, m_{2}\right)
$$

Далее рассуждение аналогично предыдущему. Заметим, что $c\left(T_{v}, 1\right)=V\left(T_{v}\right)$. Мы хотим сравнить $c\left(T_{v}, m ; i\right)$ и $c\left(T_{v}, m ; i+1\right)$, для этого итерируем предыдущее рекуррентное соотношение для $c\left(T_{v}, m ; i+1\right)$ до конца, т.е. получим сумму слагаемых $B_{s}^{i+1}$, в каждом из которых все множители будут иметь вид $c\left(T_{v^{\prime}}, 1\right)$ для некоторых $v^{\prime}$. Итерационному процессу для $c\left(T_{v}, m ; i+1\right)$ соответствует аналогичньй процесс для $c\left(T_{v}, m ; i\right)$, и в силу этого каждому слагаемому $B_{s}^{i+1}$ будет соответствовать слагаемое $B_{s}^{i}$ в разложении $c\left(T_{v}, m ; i\right)$. В этом слагаемом один из множителей будет $c\left(T_{v^{\prime}}, 2\right)$ вместо $c\left(T_{v^{\prime}}, 1\right)$ в слагаемом $B_{s}^{i+1}$. Поэтому, как и раньше, $\frac{c\left(T_{v}, m\right)}{c\left(T_{v}, m-1\right)} \gtrsim 2$. Откуда и следуют оценки, равномерные по голым деревьям. Влияние гранищ здесь также экспоненщиально мало.

Повторяя эти рассуждения для двухчастичных функций, можно доказать следующую теорему.

ТеОРема 12. При $V \rightarrow \infty$ для любъх двух вериин $v_{1}, v_{2}$ c $l\left(v_{i}\right) \rightarrow \infty$ имеет место

$$
\left|\left\langle q_{v_{1}} q_{v_{2}}\right\rangle-\left\langle q_{v_{1}}\right\rangle\left\langle q_{v_{2}}\right\rangle\right|<c \exp \left(-\alpha\left|v_{1}-v_{2}\right|\right)
$$




\section{7. Внутренняя динамика}

В предыдущих разделах рассматривалась динамика, где за один шаг возможны изменения либо только в окрестности корневой вершины, либо только на границе триангулящии диска. Здесь мы рассмотрим динамику, где изменения возможны в любом месте комплекса.

Термодинамический предел локальных процессов. Для заданной стохастической динамики мы называем локальным процессом процесс $q_{v}(t)$ - число ребер в момент $t$, инцидентных заданной вершине $v$. Предполагается, что эта вершина, выделенная в момент $t=0$, не исчезает. Мы дадим два примера интересного поведения таких локальных процессов.

Первый пример будет для движений Гросса-Варстеда. Заметим, что их можно ввести не только для симплициальных комплексов, но также и для других введенных вьше классов комплексов с клетками-треугольниками. Назовем звездой $\operatorname{St}(v)$ вершины $v$ ребра и треугольники, инцидентные к ней вместе с их границами.

Рассмотрим GV-движения типа 1 и 2, а также движение, обратное к движению типа 2, и рассмотрим цепь Маркова с множеством состояний - комплексами класса $\mathbf{A}$, в которых все клетки являются треугольниками. Скачки определяются интенсивностями $\lambda_{i}, i=1,2$, и $\mu$ этих движений соответственно для каждой пары смежных треугольников в случае движения типа 1 , для каждого треугольника в случае движения типа 2 и для звезды каждой вершины порядка 3 в случае движения, обратного к движению типа 2.

Если $\lambda_{2}=\mu=0$, то $V, L, N$ - инварианты. Пусть $A^{\prime} \subset A(N, L)$ - неприводимые компоненты в множестве комлексов с данными $N$ и $L$, положим $C\left(A^{\prime}\right)=\left|A^{\prime}\right|$. Сформулируем следующую лемму без доказательства.

Лемма 13. Если $\lambda_{2}=\mu=0, \lambda_{1}=\lambda$, то цепь Маркова на каждой $A^{\prime}$ обратима по отношению к равномерной мере. В классе симплициальньх комплексов единственная неприводимая компонента совпадает со всем классом.

Заметим однако, что первое утверждение леммы проверяется условием $\pi_{\alpha} \lambda_{\alpha \beta}=$ $\frac{\lambda}{C\left(A^{\prime}\right)}=\pi_{\beta} \lambda_{\beta \alpha}$, если $\lambda_{\beta \alpha}=\lambda_{\alpha \beta}=\lambda$.

Возьмем в момент 0 некоторьй комплекс с $N$ вершинами, выберем в нем вершину $v$ и рассмотрим случайные величины $q_{v}^{(N)}(t)$ - числа ребер в $v$ в момент $t$. Следуюший пример показывает, что пределы больших времен и больших $N$ могут быть не перестановочны, т.е.

$$
\lim _{t \rightarrow \infty} \lim _{N \rightarrow \infty} \neq \lim _{N \rightarrow \infty} \lim _{t \rightarrow \infty}
$$

для локальных величин. Таким образом, моделирование по Монте-Карло может быть обманчивьм.

ЛЕмма 14. При $N \rightarrow \infty$ предельный процесс существует и является случайным блужданием на $[3, \infty)$ с интенсивностями перехода $\lambda_{i, i+1}=\lambda_{i, i-1}=\lambda i$. Тогда

В то же время

$$
\lim _{t \rightarrow \infty} \lim _{N \rightarrow \infty} P\left(q_{v}^{(N)}(t)=k\right)=0 .
$$

$$
\lim _{N \rightarrow \infty} \lim _{t \rightarrow \infty} P\left(q_{v}^{(N)}(t)=k\right) \neq 0 .
$$


ДокАЗАТЕльСтво. Имеем $L=3 N / 2, V=(N+4) / 2$ и $\lim _{N \rightarrow \infty} \lim _{t \rightarrow \infty} q_{v}^{(N)}(t)=$ $L / V=3$. В то же время заметим, что для фиксированного $N$ процесс $q_{v}^{(N)}(t)$ является марковским с множеством состояний $3, \ldots, N$ и интенсивностями переходов $\lambda_{i, i+1}=\lambda_{i, i-1}=\lambda i$ из состояния $i$. Действительно, каждое ребро, инцидентное c $v$, может быть изменено на трансверсальное, кроме того, для каждого треугольника, содержащего $v$, его ребро, не содержашее $v$, может быть удалено некоторым GV-движением, что даст одно дополнительное ребро, инцидентное $v$. Предельное случайное блуждание будет нулевым возвратным. Лемма доказана.

Заметим, что мы сушественно использовали вид звезды вершины, устроенной как зонтик с центром в вершине $v$.

Рассмотрим теперь цепь Маркова, в которой единственньми переходами будут движения Александера, А-движения. Каждая вершина $i$ конкурирует за право сделать А-движение, иначе говоря, из нее вьпускаются два пуассоновских процесса с интенсивностями $\lambda$ и $\mu$ соответственно, и первое событие в одной из вершин производит A-движение. Если событие соответствует первому процессу, то выбирается (с вероятностью $q_{i}^{-1}$ ) одно из ребер на границе $\mathrm{St}(i)$ и производится А-движение, соответствуюшее этому ребру. Если же второму, то случайно выбирается одна из вершин $v$ степени 4 на гранище $\partial \mathrm{St}(i)$, выбирается одно из двух возможных обратных А-движений, которое и производится. Если нет вершин степени 4 на $\partial \operatorname{St}(i)$, то никаких действий не производится. Далее процесс начинается сначала.

Заметим, что появившаяся вершина $i$ может в дальнейшем исчезнуть. Пусть $t(i)-$ время, когда вершина $i$ появилась.

ТеОРема 13. Если $\lambda>\mu$, то $q_{i}(t) \rightarrow \infty$ с положительной вероятностью. Eсли $\lambda<\mu$, то вериина исчезает п.н. и $\mathrm{E} q_{i}(t)$ равномерно ограничено.

ДокАЗАТЕЛЬСТво. Пусть для каждой вершины $v a(t)=a_{v}(t)-$ число вершин $j$ в $\partial \operatorname{St}(v)$ с $q_{j}=4$, пусть $b(t)=q_{v}(t)-a(t)$. Фиксируем вершину $i$. Если $v \in \partial \operatorname{St}(i)$, то обозначим $v+1$ следуюшую вершину на $\partial \mathrm{St}(i)$ по часовой стрелке.

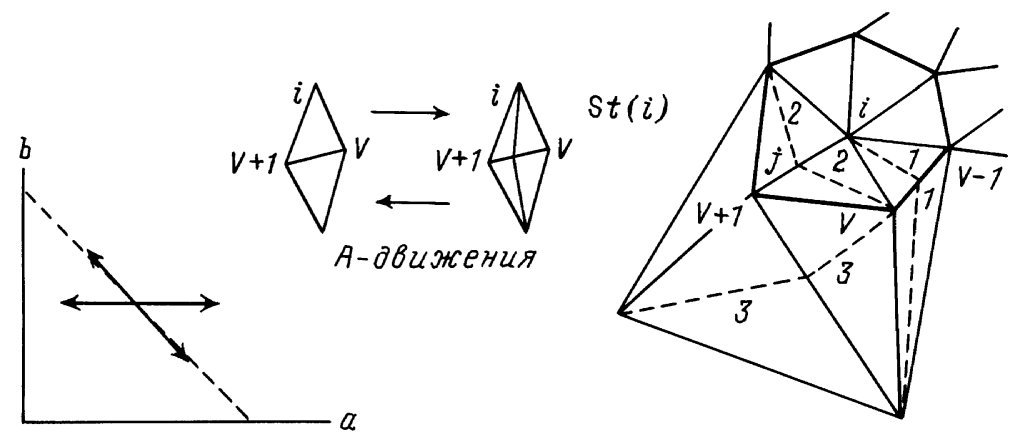

Рис. 10. Доказательство теоремы

Рассмотрим процесс $\left(a_{i}(t), b_{i}(t)\right)$ и для данной конфипурации вне $\mathrm{St}(i)$ запишем возможные инфинитезимальные скачки в $\mathbb{Z}_{+}^{2}$. Для прямого и обратного А-движения есть только три возможности.

1. Два ребра (отмеченные 1 на рис. 10) появляются на некотором ребре $((v-1, v)$ 
на рис. 10). Таким образом, здесь переход есть $a, b \rightarrow a+1, b$ и имеет интенсивность $2 \lambda \frac{q}{q}=2 \lambda$. Здесь и далее множитель 2 появляется, так как одно и то же движение может порождаться также от противоположной вершины. Обратное движение $a, b \rightarrow a, b+1$ имеет интенсивность $2 \mu \frac{a}{a}=2 \mu$.

2. Это движение порождается вершиной $v \in \partial \mathrm{St}(i)$ (пунктирные ребра 2 на рис. 10), новая вершина появляется на ребре $(i, v+1)$. Это дает изменение вектора $(a, b)$, только если $q_{v+1} \neq 4$. Таким образом, $a, b \rightarrow a+1, b-1 \mathrm{c}$ интенсивностью $2 \lambda a \frac{b}{q_{v}}$. Обратное движение дает скачок $a, b \rightarrow a-1, b \mathrm{c}$ интенсивностью $2 \alpha \mu \frac{a}{a_{v}}$.

3. Следуюшее движение также производится вершиной $v$ (ребра 3 на рис. 10 ). $q_{v}$ может перейти как $4 \rightarrow 3,5 \rightarrow 4$.

Фактически нам не нужны интенсивности для 2 и $3:$ заметим только, что эти скачки сохраняют $q_{i}=a+b$. Пусть сначала $\mu>\lambda$. Тогда вложенньй процесс $f(n)=q_{i}\left(t_{n}\right)=$ $a\left(t_{n}\right)+b\left(t_{n}\right)$, где $t_{n}$ моменты скачков, удовлетворяет неравенству

$$
\mathrm{E}(f(n+1) \mid f(n))<f(n)-\varepsilon
$$

для некоторого $\varepsilon>0$. Посредством мартингальной техники (см., например, [32]) мы получаем доказательство. Для $\mu<\lambda$ мы имеем

$$
\mathrm{E}(f(n+1) \mid f(n))>f(n)+\varepsilon,
$$

и применим аналогичный метод.

Кажется вероятным, что если $\lambda<\mu$, то для всех последовательностей $t(i) \rightarrow \infty$ процесс $q_{i}(t)$ стремится к некоторому распределению, если $s=t-t(i)$ фиксировано. Если $\lambda \ll \mu$, то это может быть доказано строго. Наоборот, в критическом случае $\mu=\lambda$ случайные величины $q_{i}(t)$ флуктуируют как в броуновском движении. Сравнение с результатами предыдущих разделов дает аргумент, что мы не получаем необходимой инвариантной меры при такой динамике.

\section{СПИСОК ЛИТЕРАТУРЫ}

[1] Regge T. General Relativity without coordinates // Nuovo Cimento. 1961. V. 19. № 3. P. 558-571.

[2] Misner Ch., Thorne K., Wheeler J. Gravitation. San Francisco: W.H. Freeman and Comp., 1973.

[3] Hawking S. Spacetime foam // Nuclear Phys. B. 1978. V. 144. P. 349-361.

[4] Kazakov V., Staudacher M., Wynter Th. Exact solution of discrete two-dimensional $R^{2}$ gravity // Nuclear Phys. B. 1996. V. 471. P. 309-333.

[5] Rovelli C. String, Loops and others: a critical survey of approaches to quantum theory // Preprint. gr-qc/9803024, 1998.

[6] Малышев В. А. Случайные грамматики // УМН. 1998. Т. 53. № 2. С. 107-134.

[7] Malyshev V.A. Stochastic evolution via graph grammars // INRIA Preprint № 3380, March 1998; see also Discrete Mathematics. 1998. № 2.

[8] Penrose R. The Emperor's New Mind. Concerning Computers, Minds and the laws of physics. Oxford: Oxford Univ. Press, 1990. 
[9] Ambjorn J. Quantization of Geometry. Les Houches Lectures, 1994.

[10] Малышев В.А. Взаимодействуюшие цепочки символов // УМН. 1997. Т. 52. № 2. C. 59-86.

[11] Gajrat A., Iasnogorodski R., Malyshev V. Null-recurrent string // Markov Process Relat. Fields. 1996. V. 2. № 3. P. 427-460.

[12] Фоменко А. Т., Фукс Б. Д. Курс гомотопической топологии. М.: Наука, 1989.

[13] Gross J., Tucker Th. Topological Graph Theory. New York: Wiley, 1987.

[14] Sternberg Sh. Lectures on Differential Geometry. Englewood Cliffs: Prentice-Hall, 1964.

[15] Alexander J. The Combinatorial Theory of Complexes // Ann. of Math. 1930. V. 31. P. 292-320.

[16] Gross M., Varsted S. Elementary moves and ergodicity in D-dimensional simplicial quantum gravity // Nuclear Phys. B. 1992. V. 378. P. 367-380.

[17] Tutte W. On the enumeration of convex polyhedra // J. Combin. Theory. Ser. B. 1980. V. 28. P. $105-126$.

[18] Wormald N. On the number of planar maps // Canad. J. Math. 1981. V. 23. P. 1-11.

[19] Wormald N. Counting unrooted planar maps // Discrete Math. 1981. V. 36. P. 205-225.

[20] Richmond L., Wormald N. Almost all maps are asymmetric // J. Combin. Theory. Ser. B. 1995. V. 63. P. 1-7.

[21] Zamolodchikov A. B. On the entropy of random surface // Phys. Lett. B. 1982. V. 117. P. $87-90$.

[22] Goulden I., Jackson D. Combinatorial Enumeration. New York: Wiley, 1983.

[23] Tutte W. The enumerative theory of planar maps // A Survey of Combinatorial Theory, Simpos. Colorado State Univ., Colorado, 1971 / ed. J. Srivastava et al: North-Holland, 1973. P. 437-448.

[24] Tutte W. A census of planar triangulations // Canad. J. Math. 1962. V. 14. P. 21-38.

[25] Tutte W. What is a map? // New Directions in the Theory of Graphs. Proc. Third Ann Arbor Conf., Univ. Michigan, 1971 / ed. F. Harary, 1973. P. 309-325.

[26] Bender E., Richmond L. A survey of the asymptotic behaviour of maps // J. Combin. Theory. Ser. B. 1986. V. 40. P. 297-329.

[27] Pastur L. A. Spectral and probabilistic aspects of random matrix models // Algebraic and Geometric Methods in Mathematical Physics / ed. A. Boutet de Monvel, V. A. Marchenko. Dordrecht: Kluwer, 1996. P. 205-242.

[28] Малышев В. А., Минлос Р. А. Гиббсовские случайные поля. М.: Наука, 1985.

[29] Bessis D., Itzykson C., Zuber J. Quantum Field Theory Techniques in Graphical Enumeration // Adv. Appl. Math. 1980. V. 1. P. 109-157.

[30] Athreya K., Ney P. Branching Processes. New York: Springer-Verlag, 1972.

[31] Lang S. Algebra. Reading, MA: Addison-Wesley, 1965.

[32] Fayolle G., Malyshev V., Menshikov M. Constructive Theory of Countable Markov Chains. Cambridge: Cambridge Univ. Press, 1995. 